\title{
Survey of Research for Performance Measurement of Mobile Manipulators
}

\author{
Roger Bostelman ${ }^{1,2}$, Tsai Hong ${ }^{1}$, and Jeremy Marvel $^{1}$ \\ ${ }^{1}$ National Institute of Standards and Technology, \\ Gaithersburg, MD 20899 \\ ${ }^{2}$ IEM, Le2i, Université de Bourgogne, \\ BP 47870, 21078 Dijon, France \\ roger.bostelman@nist.gov \\ tsai.hong@nist.gov \\ jeremy.marvel@nist.gov
}

\begin{abstract}
This survey provides the basis for developing research in the area of mobile manipulator performance measurement, an area that has relatively few research articles when compared to other mobile manipulator research areas. The survey provides a literature review of mobile manipulator research with examples of experimental applications. The survey also provides an extensive list of planning and control references as this has been the major research focus for mobile manipulators which factors into performance measurement of the system. The survey then reviews performance metrics considered for mobile robots, robot arms, and mobile manipulators and the systems that measure their performance, including machine tool measurement systems through dynamic motion tracking systems. Lastly, the survey includes a section on research that has occurred for performance measurement of robots, mobile robots, and mobile manipulators beginning with calibration, standards, and mobile manipulator artifacts that are being considered for evaluation of mobile manipulator performance.
\end{abstract}

Key words: mobile manipulator; performance measurement; standard; survey; test method.

Accepted: June 14, 2016

Published: June 30, 2016

http://dx.doi.org/10.6028/jres.121.015

\section{Introduction}

The importance and popularity of research toward mobile robots and mobile manipulators (robotic arms on mobile bases) has grown in recent years [1]. This emphasis on research and development has also spiked in recent years due to advances in technology that have enabled broad use of automation and robotics while simultaneously reducing costs. In general, mobile manipulators have been more recently researched and are now becoming commercial tools for industrial use [2-4]. In research, considerations have focused on the coordination of movements of the robot and the base since redundant degrees of freedom (DoF) are created by adding the moving base. Measurements of mobile manipulators performing standard tasks (poses and motions) are non-existent except for simply ensuring that the task has been more or less completed. Although mobile manipulators have been researched for use in many areas including marine [5], undersea [6], agriculture [7], and space [8] applications, this paper mainly focuses on manufacturing and industrial applications.

An example mobile manipulator consists of a six DoF robot arm mounted onboard a wheeled base (e.g., automatic guided vehicle, AGV, or mobile robot) with two translational and one rotational DoF in the horizontal plane for a total of nine DoF [9]. Some mobile manipulators have more or fewer DoF and may also be equipped with vertical axis motion control of the robot arm base. Holonomic and non-holonomic are frequently used terms throughout mobile robot research to describe system kinematics. Mobile manipulator control can range from manual teleoperation to full autonomy (i.e., an operator or facility 
management system controls the mobile manipulator using typically high level remote commands to the onboard controller with no direct operator interface).

Metrics for safety and performance of mobile manipulators include many areas, such as: safe operation, task completion, time to complete the task, quality, and quantity of tasks completed. Inherent in "quality" and "quantity" are the mobile manipulator accuracy and repeatability, respectively. These metrics not only include task completion but also focus on how well and how many times the task was completed.

Prior to industrial acceptance and standards development for mobile manipulators, users of these new systems will expect manufacturers to provide real performance data to guide their procurement and assure suitability for given application tasks. Robot performance measurements typically include the difference in position and orientation, or pose, of the end of arm tooling from the commanded robot pose. Ground truth measurement using motion tracking systems provides relatively accurate robot joint, segment, or tool point position information, enabling comparisons with the commanded pose. Review of robot, mobile robot, and mobile manipulator performance measurement research shows this as being a relatively new technique for performance evaluation within the research community.

The motivation and objective of the survey are to provide a current state-of-the-art in mobile manipulator research and performance measurements for these systems. The target audience of the article is robotics researchers, users, manufacturers, and standards developers of mobile manipulators.

The survey provides a literature review of mobile manipulator research with examples of experimental applications. The survey also provides an extensive list of planning and control references as this has been the major research focus for mobile manipulators which factors into performance measurement of the system. The survey then reviews performance metrics considered for mobile robots, robot arms, and mobile manipulators and the systems that measure their performance. Emphasis is placed on tracking systems for dynamic measurements as needed for mobile manipulators. Lastly, the survey includes a section on performance measurement research for robots, mobile robots, and mobile manipulators, beginning with calibration, standards, and mobile manipulator artifacts being considered for evaluation of mobile manipulator performance. However, this survey does not include grasping and in-hand manipulation of objects.

\section{Mobile Manipulator Research}

Expert researchers reported in 2005: "Autonomous mobile manipulation is a relatively young discipline within robotics." [10] As will be shown in this survey, much research on mobile manipulation occurred over the last 20 years and much of the research is task specific. The United States (U.S.) National Science Foundation (NSF)/National Aeronautics and Space Administration (NASA) Workshop on Autonomous Mobile Manipulation wrote that mobile manipulation combines a wide variety of research areas, ranging from force control to mechanism design to computer vision. Workshop recommendations for U.S. research laboratories included creating technological foci in the area of dexterous manipulation and physical interaction, among other critical areas. Programmatic recommendations included development of a simple integrated mobile manipulator aimed at a much lower price point so that it could be widely disseminated.

Mobile manipulators are capable of moving about — and interacting mechanically with —environments, and may be designed specifically to assist human beings in factories and in homes. The Introduction (Sec. 1) references examples of commercial mobile manipulators [2-4, 7] developed and being used for industrial purposes. An extensive literature review of the research leading to commercial mobile manipulators is provided in this section. As a precursor to mobile manipulator research, a literature review of mobile robot research was performed [1], which also includes a few examples of research on mobile manipulators. An earlier work by Bogh et al. [11] at Aalborg University, Denmark, provided a timeline of mobile manipulators that spans 30 years (see Fig. 1). The timeline shows relatively lightweight manipulators on a variety of mobile robots used for research, and effectively concludes with the authors' own platform ${ }^{1}$

\footnotetext{
${ }^{1}$ Commercial equipment and materials are identified in order to adequately specify certain procedures. In no case does such identification imply recommendation or endorsement by the National Institute of Standards and Technology, nor does it imply that the materials or equipment identified are necessarily the best available for the purpose.
} 


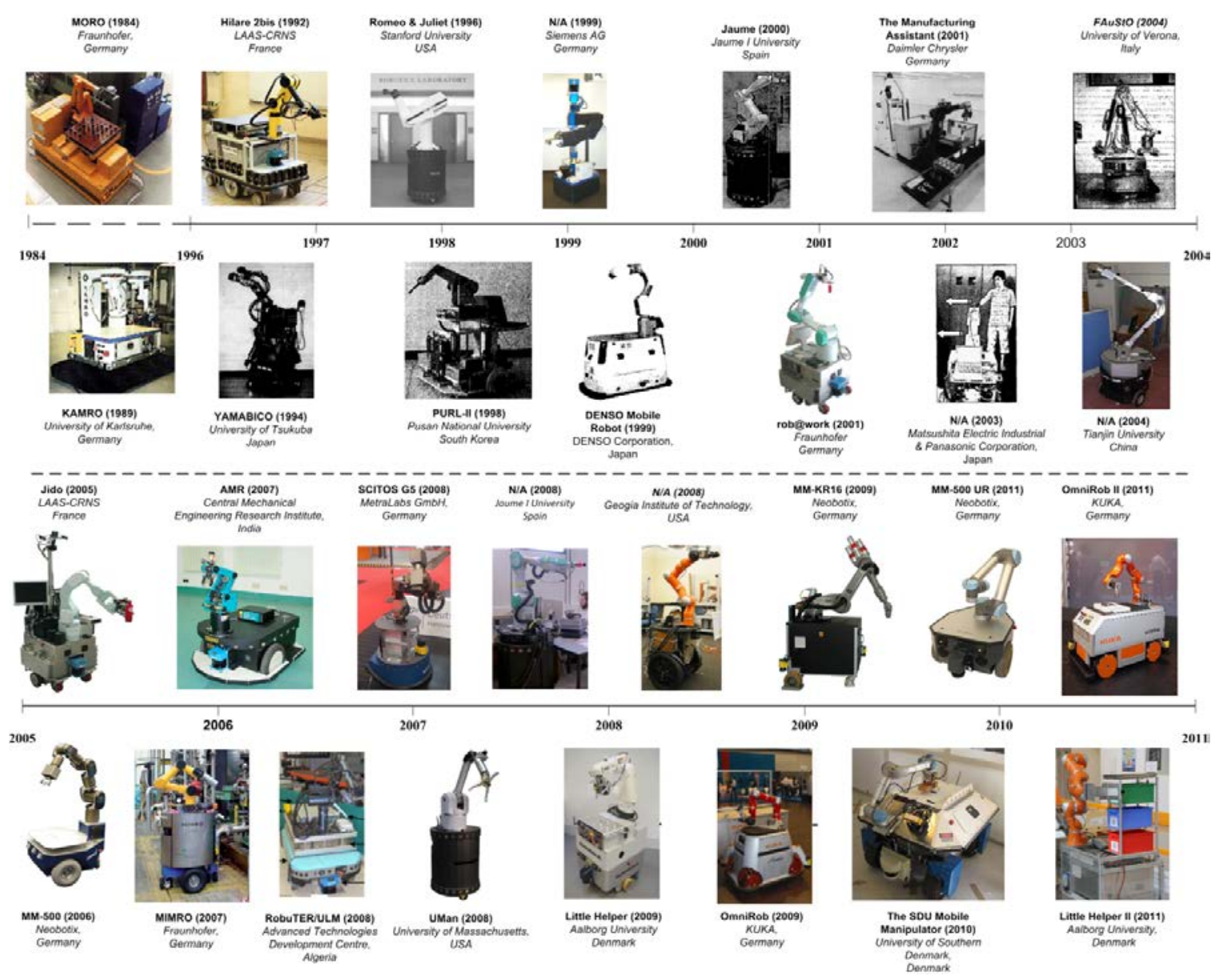

Fig. 1. Timeline of mobile manipulator development. (Photo courtesy of MTECH, Aalborg University, Denmark [11]).

designed as a proof-of-concept for general-purpose applications (such as material handling) within an industrial environment.

Many mobile manipulator applications are suggested throughout the research and discussed within this survey, for example:

- $\quad$ Processing or assembly of large-scale parts such as wind turbine blades or towers

- Surface processing (sanding, coating removal, or painting) of large systems such as aircraft or ships

- Welding of large-scale parts and components

- Large-scale rapid prototyping

- Conveyor tending

- Material handling

- $\quad$ Flexible manufacturing

This section provides an in-depth literature mobile manipulator research review divided into sections on: experimental applications and planning and control. Because of the major research efforts in mobile manipulator planning and control, this section is further divided into subsections on: unified motion, trajectory planning, configuration optimization, multiple tasks, stability, obstacle avoidance, outdoor use, and miscellaneous areas. 


\subsection{Experimental Applications}

Through direct experimental applications, several researchers have improved the capabilities of mobile manipulators solving potential future large volume robot arm applications. Hamner et al. [12] developed an autonomous mobile manipulator system that was demonstrated experimentally to achieve "peg-in-hole" type of insertion assembly tasks. The system overcame inherent system uncertainties and exceptions by using control strategies that employ coordinated control, combined visual and force servoing, and incorporated reactive task control. A force-controlled, spiral search was used to overcome the positioning uncertainty of the system relative to the assembly environment. Researchers at Aalborg University described in [13] and demonstrated in [14] how their mobile manipulators initially calibrated to a fixture and then performed peg-in-hole assembly of a rotary shaft for a pump. Flannigan et al. [15] developed a mobile manipulator system that utilized remote position sensing of the end-effector to accurately operate over a large work envelope, for example an aircraft wing or fuselage. The goal was to utilize an off-theshelf, industrial, seven DoF robot arm, an omni-directional mobile platform, and a metrology system. The metrology system tracked multiple objects within a large work space with relatively high accuracy $(200 \mu \mathrm{m})$ and in real-time. Chen et al. [16] designed a hierarchical intelligent controller using a neural network for the coordinated control of a mobile manipulator that identified and remedied leakage points of jugs filled with dangerous chemicals. The controller consisted of decision, processing, and execution levels.

Peterson et al. [17] and Nagatani et al. [18] developed force/torque control of a mobile manipulator to open a door. Alternatively, Chitta et al. [19] showed how to overcome the high-dimensionality of the planning problem by identifying a graph-based representation that was small enough for efficient planning yet contained coordinated arm and base motions to open varying type doors. Pin and Culioli [20] studied a particular aspect of the mobile manipulator kinematic redundancy resolution for material handling tasks. Specifically, they studied the system's utility to optimize its position and configuration during task commutations to handle changes in task requirements or constraints. Basic optimization schemes were developed for cases when load and position constraints are applied at the end-effector.

Various optimization criteria have been investigated for task requirements including obstacle avoidance, maneuverability, and several torque functions. Similarly, although for human assistance, Advait and Kemp [21] developed EL-E (see Fig. 2). Once provided with a three-dimensional (3D) location via brief illumination with a laser pointer, the robot autonomously approached the location and then either grasped the nearest object or placed an object.

Agah and Tanie [22] discussed control issues for a service mobile manipulator delivering and handing objects to a human. In [23], Tomizawa et al. described a system which used a mobile manipulator as a remotely teleoperated tool to help humans browse books located in a library via the Internet. A voiceguided mobile manipulator was presented by Bort and Pobil [24]. The user could interact verbally with the system to help it localize, identify, database, and pick up objects.

Holmberg and Khatib [25] designed and developed a powered caster vehicle (PCV) which provided the desired smooth, accurate motion and coordination with an onboard manipulator, a holonomic vibration-free wheel system that can be dynamically controlled. They proposed a new approach for modeling and controlling the dynamics of this parallel redundant system.

Dual manipulators have also been researched, mainly for material handling. In 1996, Khatib et al. [26, 27] developed multiple mobile manipulation systems and the basic models and methodologies for their analysis and control. They extended four fixed base manipulation methodologies to mobile manipulation systems, called Stanford Assistant Mobile Manipulators (SAMM). The methodologies included: "1) the Operational Space Formulation for task-oriented robot motion and force control; 2) the Dexterous Dynamic Coordination of Macro/Mini structures for increased mechanical bandwidth of robot systems; 3) the Augmented Object Model for the manipulation of objects in a robot system with multiple arms; and 4) the Virtual Linkage Model for the characterization and control of internal forces in a multi-arm system." Figure 3 shows a graphical depiction of the SAMM carrying a pipe. 


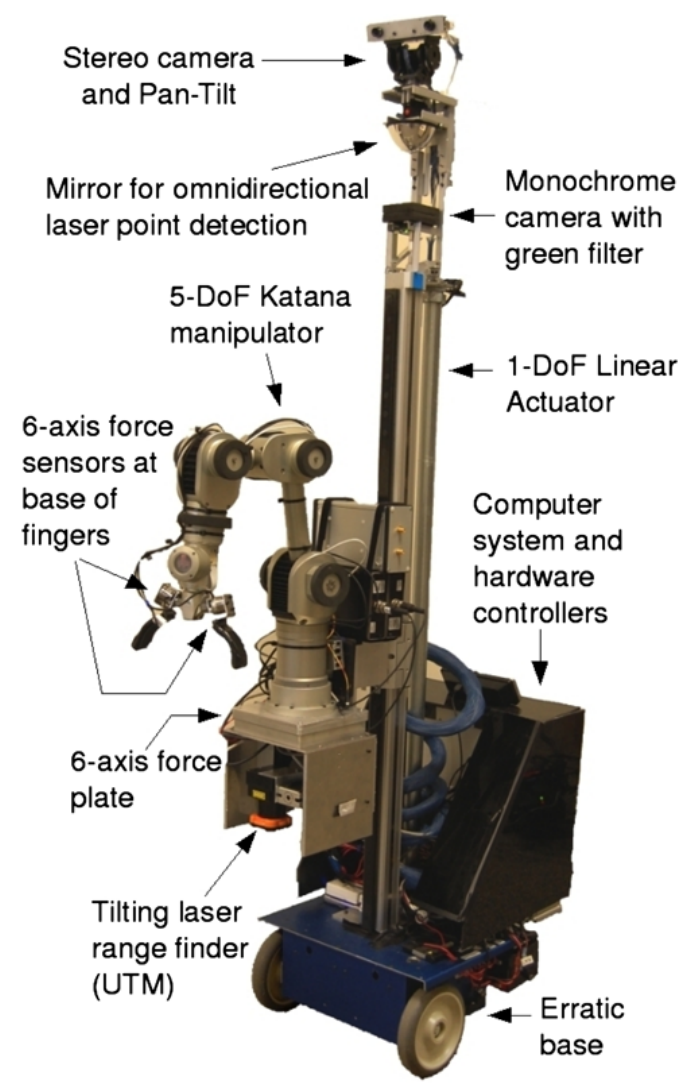

Fig. 2. EL-E mobile manipulator [22].

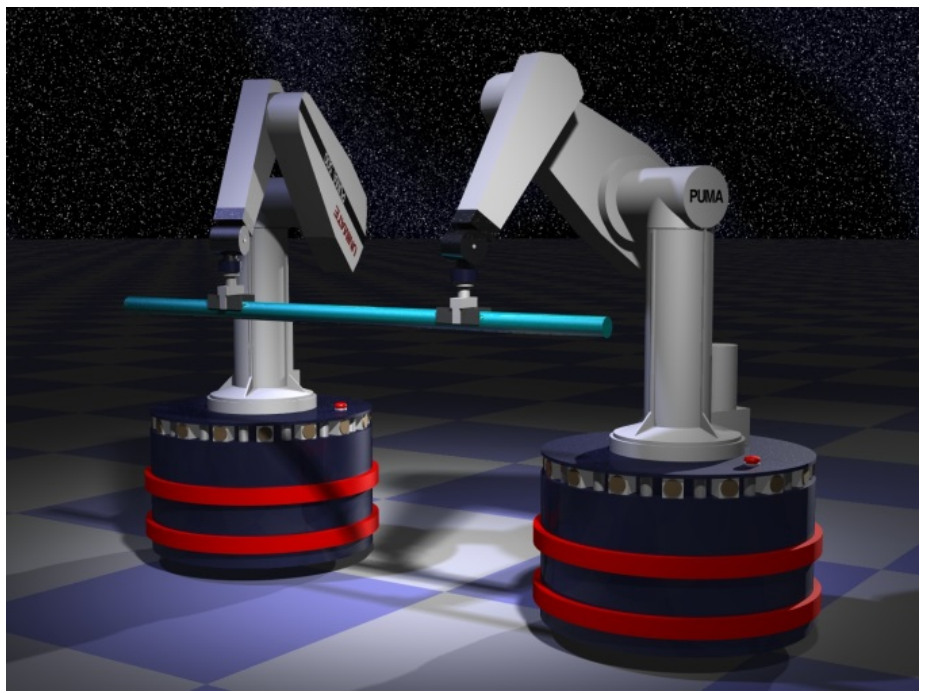

Fig. 3. Graphical depiction of the Stanford Assistant Mobile Manipulators (SAMM) carrying a pipe [26]. 
Cooperating control (a derivation of force control) of multiple mobile manipulators was later studied by Osumi et al. [28] and Sugar and Kumar [29]. In 2005, Stroupe et al. [30], demonstrated a behavior-based system in which two mobile-base robots perform assembly tasks. One robot served as a master and the other as a slave in the assembly of beams into a structure. Stereo vision was used to locate fiducial marks on the components and force-torque sensing was used during transportation and assembly.

Beyond the laboratory, Madsen et al. [31] evaluated two autonomous mobile manipulators in a realworld, industrial pump, manufacturing environment. The objective was to integrate the mobile manipulators with existing equipment to increase the technology-readiness-level for industrial use. The two robots worked together to produce rotors over a period of 10 days and were set up in less than a day. The experiment included workspace adaptation, safety regulations, rapid robot instruction and running production. Not included in the Fig. 1 timetable is the Meka M1 Mobile Manipulator [3], pictured in Fig. 4 which also included a vertical axis for the manipulator. The Meka M1 is being further developed by Alphabet and includes a humanoid system equipped with two compliant manipulators with 6-axis forcetorque sensors at the wrist, compliant grippers, a "head" with a 3D sensor, and an omnidirectional wheel base.

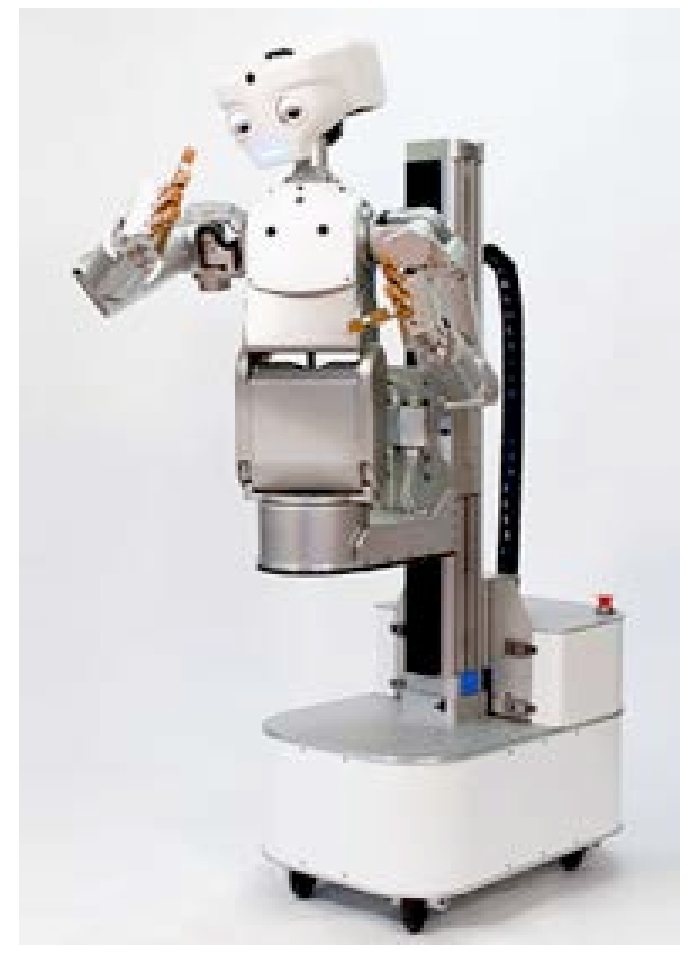

Fig. 4. Meka M1 Mobile Manipulator [3].

Design of mobile manipulators has also been researched for specific or generic use. Towards design of a mobile manipulator for a specific application, namely automated highway construction and maintenance, Gardner and Velinsky [32] described a systematic, unified, kinematic analysis for manipulator arms mounted to mobile platforms. The differential kinematics for the combined system was used, along with an extended definition of manipulability, to generate a design tool for this class of systems. An example was presented in which a three DoF anthropomorphic manipulator was mounted on a platform powered by two independent drive wheels. Scaled manipulability ellipses were used to visualize the effect of manipulator mounting position on the overall mobility of the system. 


\subsection{Planning and Control}

Most of mobile manipulator research has been in planning and control due to the redundant DoF, such as the stability of the system with a moving manipulator onboard. While complicating planning and control, this kinematic redundancy is desirable since it allows mobile manipulators to operate under many modes of motion and to perform a wide variety of tasks. The survey of planning and control is, therefore, divided into several areas where research was published, including unified motion of the manipulator with the base, trajectory planning and coordinated control of the system, the system performing multiple tasks, stability, obstacle avoidance of the manipulator combined with the base, the system use outdoors, and other miscellaneous mobile manipulator research.

\subsubsection{Unified Motion}

To plan a mobile manipulator's motion, some researchers considered the onboard manipulator as extra joints of the mobile base, where the locomotion controller is part of the manipulator controller. However, it is difficult to implement both controllers as one since the controllers are typically manufactured by two different companies. In 2000, Papadopoulos and Poulakakis [33] described a planning and control methodology for mobile manipulators, allowing them to follow simultaneously-desired end-effector and platform trajectories without violating the non-holonomic constraints. In 2002, Kim et al. [34] formulated a combined approach for the mobile manipulator mobility and manipulation using a redundant scheme which used zero moment point. In the same year, Nagatani et al. [35] developed a path planning algorithm where the locomotion controller was independent from the manipulator controller, and cooperative motion occurred using communication between controllers. Further, in 2006, Katz, et al. [36] considered endeffector-centric, integrated mobility with manipulation, or simply, tool-point control. The combination resulted in an experimental platform that allowed researchers to focus only on end-effector behavior without having to worry about the motion of the remaining DoF. Mailah et al. [37] proposed in 2006 the resolved acceleration control (RAC) and proportional-integral active force control (PIAFC) as an approach for the robust mobile manipulator motion control. The concept utilized a differentially-driven, wheeled, mobile platform with an onboard two-link planar arm. The study emphasized the integrated kinematic and dynamic control strategy in which the RAC was used to manipulate the kinematic component while the PIAFC compensated for dynamic effects, including disturbances and uncertainties.

\subsubsection{Trajectory Planning}

Several researchers have studied trajectory planning. In 1997, Chen and Zalzala [38] developed an approach for the modeling and motion trajectory planning of a mobile manipulator system with a nonholonomic constraint. Tanner and Kyriakopoulos [39], in 2001, used Kane's dynamic equations. Mohri et al. [40], also in 2001, developed a trajectory planning method to optimally control the combined system of a mobile manipulator with the end-effector's specified path. Shin et al. [41], in 2003, presented a motion planning method for mobile manipulators where the base was moved to discrete poses from which the manipulator could be deployed to cover a prescribed trajectory. Finally, in 2011, Tang et al. [42] presented differential-flatness-based, integrated, point-to-point trajectory planning and control.

\subsubsection{Configuration Optimization}

In 1992, Yamamoto and Yun [43] studied a planning and control algorithm for a mobile manipulator so that the manipulator was always positioned at the preferred configurations measured by its manipulability. Simulation results initially proved the intended algorithm result followed by implementation and verification on a real mobile manipulator system. In 1997, Chen and Zalzala [44] described a genetic algorithm approach to multi-criteria motion planning of mobile manipulator systems. Least-torque norm, manipulability, torque-distribution, and obstacle avoidance were considered for multi-criteria, mobile manipulator position and configuration optimization. The emphasis of the simulated study was placed on 
using genetic algorithms to search for global, optimal solutions and solve the minimax problem for manipulator torque distribution.

\subsubsection{Multiple Tasks}

In 1994, Pin et al. [45] focused on minimax planning the positions and configurations in which the system needed to be at task commutation in order to assure that it could properly initiate the next task to be performed. An implementation of the algorithms was performed using the HERMIES-III mobile manipulator. In 1997, Lee and Cho [46] proposed a new motion planning method for mobile manipulators to sequentially execute multiple tasks where, the final simulated configuration of each task was the initial configuration of the subsequent task.

\subsubsection{Stability}

As mentioned in the previous section, stability has been a large area for mobile manipulator research. As a mobile manipulator moves at high speeds and/or if the manipulator carries a non-centric load, the manipulator can dynamically disturb the vehicle, even causing a vehicle to tip over. Mobile manipulators operating in industrial or field environments will be required to apply forces, manipulate loads, and to perform such tasks on uneven terrain which may cause the system to approach, or reach, a dangerous tip over instability. To avoid tip over in an automatic system, or to provide a human operator with an indication of proximity to tip-over, a variety of control methods have been researched.

In 1989, Dubowsky and Vance [47] presented a planning method for mobile manipulators which insured that dynamic disturbances did not exceed the capabilities of the vehicle, and compromise its stability, while permitting the mobile manipulator to perform its tasks rapidly. Dubowsky et al. [48] later derived the dynamic equations of a fully spatial mobile manipulator with link flexibility. Huang et al. provided seven works [49-55] that researched stability of mobile manipulators - two examples are provided here. In 1994, Huang et al. [49] presented a control scheme for maintaining or recovering stability, called the method of zero motion path (ZMP) planning by a stability potential field, considering goal and prohibitive stability states. In 2000, Huang et al. [55] considered both stabilization and manipulation simultaneously while coordinating vehicle motion and manipulator motion. In 2001, Inoue et al. [56] verified their stability control strategy for a mobile manipulator when an external force exists and confirmed this through several experimental results. In 2003, Furuno et al. [57] presented methods of trajectory planning for a mobile manipulator with stability considerations. The proposed planning method was to generate a mobile manipulator trajectory from a given end-effector path when considering stability. Then, a dynamic model of the mobile manipulator was derived considering it as the combined system of the manipulator and the mobile platform. Zero motion point criterion was used as an index for the system stability demonstrated in simulation. In 2007, Shibata and Murakami [58] described a control strategy of mobile manipulator null space motion which used passivity-based stabilization of end-effector position. The research was verified by simulations and experiments of a two wheeled mobile manipulator.

In 1996, Papadopoulos and Rey [59] defined a measure of available stability margin and in 2000 [60], they applied the force-angle stability measure which: had a simple geometric interpretation, was easily computed, and was sensitive to changes in center of mass height. Force-angle performance measurement was compared with that of other stability margin measures using a forestry vehicle simulation. In 2006, Thibodeau et al. [61] compared whole body postural control of humanoid robots to mobile manipulators, i.e., robot arms mounted onboard mobile robots having a reduced footprint and raising the center of mass of a robot. The results suggested that for pushing, pulling, or carrying tasks, using whole body postural control could lead to higher performance by allowing a platform to apply more force to the environment.

\subsubsection{Obstacle Avoidance}

Some research, expanded from mobile robots, focused on obstacle avoidance for mobile manipulators. Since mobile manipulators can change their volumetric profile by protruding the manipulator beyond the base, the manipulator can potentially collide with obstacles that are outside of the mobile base volume. 
Referring independently to robot arms and mobile bases, in 1986, Khatib [62] presented a very well referenced and unique real-time obstacle avoidance approach for manipulators and mobile robots based on the artificial, potential-field concept. This research also has bearing to the mobile manipulator research area since the principles of collision avoidance, traditionally considered a high level planning problem, can be effectively distributed between different levels of control for both the manipulator and base.

In 1995, Yamamoto and Yun [63] developed a control method and discussed simulation results for mobile manipulators that integrated an obstacle avoidance scheme with a coordination scheme. The obstacle avoidance scheme was based on superquadric potential functions with coordination in preferred operating regions. The controller allowed a mobile manipulator to retain optimal or sub-optimal configurations while avoiding obstacles. Yamamoto and Fukuda [64] also investigated multiple, wheeled mobile manipulators coordinating with each other under a collision avoidance situation. Their earlier research in 1995 derived kinematic and dynamic manipulability for multiple mobile manipulators simultaneously transporting a single object. The later effort in 2002 extended the previous approach to a system consisting of two mobile manipulators avoiding a collision while carrying an object. The two mobile manipulators dynamically changed their configurations so that they did not collide with each other while not only maintaining the support for the object, but also keeping the arm's configuration away from singularities.

Reactive obstacle avoidance for mobile robots and sample-based motion planning for mobile manipulators were outlined in 2010 in Nowak et al. [65]. In their report, they aimed to develop a methodology for identifying and providing best practice algorithms in a range of robotics fields. The methodology found objective performance comparison measures, and finally resulted in a number of open source, best practice libraries. Suggested performance criteria are:

- $\quad$ Mission success

- $\quad$ Path length

- Time taken

- Number of collisions

- Obstacle clearance

- Robustness in narrow spaces

\subsubsection{Outdoor Use}

Researchers have studied the mobile manipulator control algorithm including dynamic motions caused by onboard manipulator motion, especially in undulating terrain [66]. In 2007, Najjaran and Goldenberg [67] presented real-time motion planning for an autonomous mobile manipulator that scanned outdoor terrain to detect landmines. In their systems, a terrain map was generated using laser and ultrasonic rangefinder measurements. A generated map of obstacles from the measurements was then used to define an obstacle-free, detector path which was verified by experiments on a prototype mine-detector robot. A significant amount of research was also done and continues in mobile manipulators for use outdoors. For example, Wells and Deguire [68] described TALON, a mobile manipulator originally developed under Defense Advanced Research Project Agency's (DARPA's) Tactical Mobile Robotics (TMR) Program to extend "the reach and capabilities of the war fighter" for explosive ordinance disposal and to counter improvised explosive devices.

In 2006, Yang and Brock [69] stated that the "autonomous execution of manipulation tasks in unstructured, dynamic environments requires the consideration of various motion constraints. Any motion performed during the manipulation task has to satisfy constraints imposed by the task itself, but also has to consider kinematic and dynamic limitations of the manipulator, avoid unpredictably moving obstacles, and observe constraints imposed by the global connectivity of the workspace. Furthermore, the unpredictability of unstructured environments requires the continuous incorporation of feedback to reliably satisfy these constraints." They presented a novel, feedback motion planning approach, called "elastic roadmap framework," which satisfied all motion constraints in autonomous mobile manipulation, when tested via simulation, as well as their respective feedback requirements. 


\subsubsection{Miscellaneous}

In 1988, Graettinger and Krogh [70] defined the acceleration radius, as opposed to the acceleration tangent, as a global index for quantifying the dynamic capabilities of manipulator-positioning systems over a continuous operating region. The authors developed a numerical algorithm to solve the unbounded acceleration issues and applied the solution to the acceleration radius concept. Manipulator design and workspace specification examples were illustrated. In 1998, Chung and Velinsky [71] presented mobile manipulator modeling and control where the equations of motion were derived using the Lagranged'Alembert formulation (i.e., considering applied forces) for a non-holonomic mobile manipulator model. The dynamic model, which considers slip of the platform's tires, was developed using the Newton-Euler method and incorporates Dugoff's tire friction model. In 2000, Watanabe et al. [72] described analysis and control for a holonomic, omnidirectional mobile manipulator, in which their platform included three lateral, orthogonal wheel assemblies and a mounted manipulator with three rotational joints, located at the platform center-of-gravity. Simulated results showed that the mobile manipulator could be controlled to retain any end-effector pose, regardless of the external, applied-force direction. In 2005, Mbede et al. [73] applied a robust, adaptive, fuzzy, reactive motion-planning algorithm for mobile manipulator navigation. The system included an incomplete mathematical robot system model and sensor data uncertainties. For the vehicle platform, they combined the advantages of a probabilistic roadmap as a planner and fuzzy navigation to allow adaptability similar to an elastic band. Stabilization, mobilization, and manipulation are all considered. In 2004, Korayem et al. [74] developed the Iterative Linear Programming (ILP) method to determine dynamic load carrying capacity of mobile manipulators. The authors presented numerical method examples that included a PUMA robot mounted on a linear-tracked base and a wheeled, mobile manipulator.

\section{Performance Metrics}

Albus [75] wrote: "A major barrier to the development of intelligent systems is the lack of metrics and quantifiable measures of performance. There cannot be a science of intelligent systems without standard units of measure. There are few benchmarks or standardized tests wherein performance can be compared.”

Benchmarks play an important role in various aspects. Some see it related to roadmaps where benchmarks may guide developments and make progress in the right directions measurable. Another important aspect is the relation to standards [76]. Benchmarks are the framework leading to standards and alternatively, standards can foster the requirement for benchmarks in a field. This section describes the metrics for mobile manipulators and research that has occurred in this area. Besides the development of a three-dimensional, statistical, evaluation framework for performance measurement of robotic systems [77] dated 1998, the majority of references date research in this area within only the last 13 years (i.e., 20032016) implying that there is a relatively new thrust in measuring robot performance.

Mobile robot navigation is a highly researched robotics challenge and one of the key capabilities for robot autonomy. Navigation techniques have become more and more reliable, but evaluation mainly focuses on individual navigation components (i.e., mapping, localization, and planning) using datasets or simulations. As an example, performance measures are often neglected in the mobile robot navigation research for applications such as: vacuuming, surface coating, and systematic foraging. In 2002, Wong et al. [78] presented two metrics for measuring performance of robot coverage (i.e., volume accessed by the robot) tasks and applied them to a real robot coverage experiment: percentage of coverage and distance traveled by the robot. The study found that the percentage of coverage is a good performance indicator if physical limitations of the robot are taken into account. On the other hand, distance traveled alone is a poor indicator because it completely ignores the amount of area (mobile base plus onboard manipulator) covered by the mobile manipulator. In 2014, Sprunk et al. [79] defined an experimental protocol to evaluate the whole navigation system, deployed in a real environment. To ensure repeatability and reproducibility of experiments, their benchmark protocol provided detailed definitions and controls for the environment dynamics (i.e., variations in different environments). They defined standardized environments and 
introduced the concept of a reference robot to allow comparison between different navigation systems at different experimentation sites.

The robotics community is seeing a growing awareness of the difficulty to compare, in a rigorous quantitative way, the many research results obtained from many different robotic application areas. Moreover, Ioan et al. [80] also wrote that "there is a lack of good benchmarks for mobile manipulator motion planning.” In 2009, Bonsignorio et al. [81] focused on the issues raised by the replication/reproducibility of results. They suggest that one aspect of these results are "a cornerstone of any scientific methodology," versus being "a basic prerequisite to compare different methods for common problems” previously proposed. In 2003, Jacoff et al. [82] discussed the development and proliferation of metrics in the form of robot test arenas that provide tangible, realistic, and challenging environments for mobile robot researchers interested in search and rescue applications and other unstructured environments. This effort may also apply to mobile manipulator performance evaluation for industrial environments.

Lampe and Chatila [83] simulated an approach to qualify robot autonomy by measuring robot performance in completing a task as compared to the robot environment complexity. Steinfeld et al. [84] attempted to identify common metrics for task-oriented, human-robot interaction (HRI). HRI can also pertain to mobile manipulators where it is expected that future smart manufacturing systems will work collaboratively with humans. More recently, specific aspects of robot measurements were considered for smart manufacturing implementation. Two examples were in mobile robots used for welding and in dynamic pose estimation, both of which are critical to also consider for mobile manipulator use. Welding requires process validation prior to use in the manufacturing process. However, there is a lack of industry standards for mechanized or robotic welding that can impede the introduction of mobile robotic welding systems in the market place. There is also a lack of generalized fitness measures that gauge the suitability of mobile robot topologies or dimensional designs to a set of tasks and can be used in the design or verification process. Canfield et al. [85] proposed such a metric and demonstrated its use in evaluating mobile robot designs for welding tasks. Similarly, Eastman et al. [86] reported that an unsolved but important problem in intelligent manufacturing is dynamic pose estimation under complex environmental conditions, i.e., tracking an object's pose as it moves in an environment with uncontrolled lighting and background. This is a central task in robotic perception, and a robust, highly-accurate solution would be of use in a number of manufacturing applications.

As an example of metrics suggested in research for mobile manipulators, Ilnicki and Zadarnowska [87] researched the problem of defining performance measures of doubly non-holonomic mobile manipulators. Results consisted of a definition of the following local performance characteristics: the dexterity ellipsoid, the local dexterity, the energy efficiency, and the motion efficiency.

Accuracy and repeatability, two common metrics considered in robotics, were described in Shiakolas et al. [88]. The factors that affect these characteristics were identified, and an error tree was developed. A new measure called "degree of influence" was established that qualitatively assesses the relative contribution of each kinematic parameter variation to the accuracy and repeatability of rigid manipulators. The developed formulation provided for easy evaluation of the degree of influence measures for rigid manipulators in either numerical or symbolic form. Based on the above references and on past research in robotics for industrial use, the authors of this survey list and define example metrics to consider for mobile manipulators. The importance of each metric is determined by the commanded task.

- time/task duration - the time for the mobile manipulator to complete a commanded task

- distance traveled - the distance that the mobile manipulator has traveled

- repeatability - the variation in measurements taken of the mobile manipulator with the same conditions per trial

- accuracy - the amount that a mobile manipulator measurement conforms to the correct value

- task completion/effectiveness - how successful the mobile manipulator is in performing a commanded task

- efficiency - the ratio of the expected mobile manipulator performance to the total performance.

- dexterity - the agile or nimble performance of a mobile manipulator

- autonomy - independent control performance of the mobile manipulator

- stability - the firmly established performance of the mobile manipulator 
- obstacle detection and avoidance - the ability of the manipulator, mobile base or combined system to perceive and avoid objects in the environment

- exploration of unknown environments - the ability of the manipulator, mobile base or combined system to perceive and navigate the environment that is not currently within the system's map

\section{Performance Measurement Systems}

Given the above metrics to consider for mobile manipulators, a survey of performance measurement systems to achieve these metrics is provided. Contact and non-contact sensors are readily available to measure various aspects of machine performance and are listed here. Each measurement system is numbered and listed in Table 1 and Table 2 as the authors suggest their usefulness in measuring performance of mobile manipulators based on the metrics listed in Sec. 3. Table 1 shows the usefulness to measure mobile manipulator performance where the systems used to measure mobile manipulator performance "may be constrained" by the measurement system and Table 2 shows the measurement "not constrained" by the measurement system. Not-constrained means only the distance, not the accuracy measurement capability which could also be affected by a minimal distance measurement capability. The " $x$ " means usefulness in measuring performance, the yellow box means that the system has little use in measurement, and the red box means the system cannot measure performance of the metric. Some limitations are also cited below for measurement systems to measure unconstrained performance of mobile manipulators.

1. Touch probes: contact measurement of positioning systems (e.g., computer numerical controlled machine backlash error measurement) [89]. [limitations may occur from touch probe measurement distance and digital vs. variable response]

2. Wireless indoor position measurement systems: three typical location estimation schemes triangulation, scene analysis, and proximity performance comparisons include: accuracy, precision, complexity, scalability, robustness [90].

3. Tools for mechanical design and performance evaluation of robots: Dynamics-based local performance evaluation of manipulator global, open- or closed-chain, and alternative dexterity, link and joint deflections, acceleration radius, elasto-static performance (i.e., robot response to applied loads), and elasto-dynamic performance (i.e., link and moments of inertia) [91].

4. Perception sensing systems: six DoF position and motion capture measurement systems, typically used to track people or other objects, are currently being used for assessing static [92] and dynamic [93] robot performance.

5. Motion tracking camera systems:

a. using passive fiducial targets [94] affixed to the subjects being tracked with submillimeter accuracy;

b. using active fiducial targets [95] affixed to the subjects being tracked;

c. two camera opto-electronics measuring system tracks the 3D location of infrared light emitting diodes (LEDs) attached to a robot with resolution of approximately $0.01 \%$ and absolute accuracy of $0.05 \%$ [96].

6. Active camera mounted on a pan/tilt platform: image mapping is used to align images of different viewpoints so that static camera motion detection could be applied. The system extracts moving edges from dynamic images [97].

7. Acoustic and inertial motion tracking systems: obtain two types of measurements, acoustic and inertial, associated with the motion of a body and used extensively for measuring performance of robot systems [98, 99].

8. Laser tracking systems (LTS): long range laser, relatively high accuracy, non-contact measurement system, used extensively to measure robot performance. System use examples are: [limitations may occur from complex or high speed motions breaking the line-of-sight beam]

a. Real-time 3D static and dynamic positioning accuracy of a robot end-effector at 12.5 $\mu \mathrm{m}-50 \mu \mathrm{m}$ and wrist orientations to within $2 \mathrm{sec}$ of $\operatorname{arc}[100]$; 
b. LTS used with a vision system for six DoF, dynamic, real-time robot measurement for high-accuracy straightness measurement, precision leveling, and absolute distance metrology [101, 102];

c. LTS with an automatic routine to reinsert calibrated parameters into robot/machine calibration tables to improve system accuracy to approximately $10 \mu \mathrm{m}$ or higher [103].

9. Multiple sensors:

a. Two lasers: static and dynamic 3D performance measurement using two laser beams to track an optical target attached to the robot end-effector, where target position coordinates are estimated using triangulation [104].

b. LTS, a laser interferometer system, and a telescopic metrology bar: an LTS and the laser interferometer system are used to measure linear errors along a linear path of motion and angular errors about axes orthogonal to the path of motion, and a telescopic metrology bar is a low-cost, high-precision tool for assessing the static and dynamic positioning performance of industrial robots [105]. [limitations may occur from complex or high speed motions breaking the line-of-sight beam]

10. Laser diode with a position-sensitive detector: a non-linear, coupled, and measurement-based dynamic system model, a Lyapunov-type controller based on the deflection feedback to measure the tip oscillations and regulate the endpoint of the flexible robot [106]. [limitations may occur for dexterity measurement since manipulator endpoint (i.e., three DoF, not six DoF) is measurement]

11. Indoor global positioning system (iGPS): multiple rotating, angled, laser scanners detect the triangulated position of transmitters with measurement accuracy of approximately $200 \mu \mathrm{m}$ [107, 108].

Table 1. Metrics and the corresponding numbered measurement systems to measure mobile manipulator performance where the systems used to measure mobile manipulator performance may be constrained by the measurement system. Boxes marked with an " $\mathrm{x}$ " indicate utility in performance measurements, while boxes highlighted in yellow or red indicate limited or no utility, respectively toward measuring the specified metrics.

\begin{tabular}{|c|c|c|c|c|c|c|c|c|c|c|c|c|c|c|c|c|}
\hline metric & 1 & 2 & 3 & 4 & $5 a$ & $5 \mathbf{b}$ & $5 c$ & 6 & 7 & $8 a$ & $8 b$ & $8 c$ & $9 a$ & $9 b$ & 10 & 11 \\
\hline (R)esearch or (C)ommerial & $\mathrm{C}$ & $\mathrm{C}$ & $\mathrm{R}$ & $\mathrm{C}$ & $\mathrm{C}$ & $\mathrm{C}$ & $\mathrm{R}$ & $\mathrm{R}$ & $\mathrm{R}$ & $\mathrm{C}$ & $\mathrm{R}$ & $\mathrm{R}$ & $\mathrm{R}$ & $\mathrm{R}$ & $\mathrm{R}$ & $\mathrm{C}$ \\
\hline time/task duration & & $x$ & $\mathrm{x}$ & $\mathrm{x}$ & $\mathrm{x}$ & $\mathrm{x}$ & $\mathrm{x}$ & $\mathrm{x}$ & $\mathrm{x}$ & $x$ & $\mathrm{x}$ & $\mathrm{x}$ & $\mathrm{x}$ & $\mathrm{x}$ & $\mathrm{x}$ & $\mathrm{x}$ \\
\hline distance traveled & $\mathrm{x}$ & $x$ & $x$ & $x$ & $x$ & $x$ & $x$ & $x$ & $x$ & $\mathrm{x}$ & $x$ & $\mathrm{x}$ & $\mathrm{x}$ & $x$ & $x$ & $x$ \\
\hline repeatability & $\mathrm{x}$ & $\mathrm{x}$ & $x$ & $\mathrm{x}$ & $x$ & $x$ & $\mathrm{x}$ & $\mathrm{x}$ & $x$ & $\mathrm{x}$ & $x$ & $\mathrm{x}$ & $\mathrm{x}$ & $\mathrm{x}$ & $x$ & $x$ \\
\hline accuracy & $\mathrm{x}$ & $\mathrm{x}$ & $\mathrm{x}$ & $\mathrm{x}$ & $\mathrm{x}$ & $\mathrm{x}$ & $\mathrm{x}$ & $\mathrm{x}$ & $\mathrm{x}$ & $\mathrm{x}$ & $\mathrm{x}$ & $\mathrm{x}$ & $\mathrm{x}$ & $\mathrm{x}$ & & $\mathrm{x}$ \\
\hline task completion/effectiveness & $\mathrm{x}$ & $x$ & $x$ & $\mathrm{x}$ & $x$ & $\mathrm{x}$ & $\mathrm{x}$ & $x$ & $x$ & $\mathrm{x}$ & $\mathrm{x}$ & $x$ & $\mathrm{x}$ & $\mathrm{x}$ & $\mathrm{x}$ & $\mathrm{x}$ \\
\hline efficiency & & $x$ & $x$ & $\mathrm{x}$ & $x$ & $x$ & $x$ & $x$ & $x$ & $\mathrm{x}$ & $x$ & $x$ & $\mathrm{x}$ & $x$ & $x$ & $x$ \\
\hline dexterity & $\mathrm{x}$ & $x$ & $x$ & $x$ & $x$ & $x$ & $x$ & $x$ & $x$ & $\mathrm{x}$ & $x$ & $x$ & $\mathrm{x}$ & & $x$ & $x$ \\
\hline autonomy & $\mathrm{x}$ & $x$ & $x$ & $x$ & $x$ & $x$ & $x$ & $x$ & $x$ & $\mathrm{x}$ & $x$ & $x$ & $\mathrm{x}$ & $x$ & $\mathrm{x}$ & $x$ \\
\hline stability & & $x$ & $x$ & $x$ & $x$ & $x$ & $x$ & $x$ & $\mathrm{x}$ & $x$ & $x$ & $x$ & $\mathrm{x}$ & $x$ & $x$ & $\mathrm{x}$ \\
\hline $\begin{array}{l}\text { obstacle detection and } \\
\text { avoidance }\end{array}$ & & $x$ & $x$ & $x$ & $\mathrm{x}$ & $x$ & $x$ & $\mathrm{x}$ & $\mathrm{x}$ & $\mathrm{x}$ & $x$ & $x$ & $x$ & & & $x$ \\
\hline $\begin{array}{l}\text { exploration of unknown } \\
\text { environments }\end{array}$ & $x$ & $x$ & $x$ & $x$ & $x$ & $x$ & $x$ & $x$ & $x$ & $x$ & $x$ & $x$ & $x$ & & & $x$ \\
\hline
\end{tabular}


Table 2. Metrics and the corresponding numbered measurement systems to measure mobile manipulator performance where the systems used to measure mobile manipulator performance are not constrained by the measurement system.

\begin{tabular}{|c|c|c|c|c|c|c|c|c|c|c|c|c|c|c|c|c|}
\hline metric & 1 & 2 & 3 & 4 & $5 a$ & $5 b$ & $5 c$ & 6 & 7 & $8 a$ & $8 \mathrm{~b}$ & $8 c$ & $9 a$ & $9 b$ & 10 & 11 \\
\hline (R)esearch or (C)ommerial & $\mathrm{C}$ & $\mathrm{C}$ & $\mathrm{R}$ & $\mathrm{C}$ & $\mathrm{C}$ & $\mathrm{C}$ & $\mathrm{R}$ & $\mathrm{R}$ & $\mathrm{R}$ & $\mathrm{C}$ & $\mathrm{R}$ & $\mathrm{R}$ & $\mathrm{R}$ & $\mathrm{R}$ & $\mathrm{R}$ & $\mathrm{C}$ \\
\hline time/task duration & & $x$ & $\mathrm{x}$ & $\mathrm{x}$ & $\mathrm{x}$ & $\mathrm{x}$ & $\mathrm{x}$ & $\mathrm{x}$ & $\mathrm{x}$ & & & & $\mathrm{x}$ & & $\mathrm{x}$ & $\mathrm{x}$ \\
\hline distance traveled & & $\mathrm{x}$ & $\mathrm{x}$ & $\mathrm{x}$ & $\mathrm{x}$ & $\mathrm{x}$ & $\mathrm{x}$ & $\mathrm{x}$ & $\mathrm{x}$ & & & & $\mathrm{x}$ & & $x$ & $\mathrm{x}$ \\
\hline repeatability & & $x$ & $\mathrm{x}$ & $\mathrm{x}$ & $\mathrm{x}$ & $\mathrm{x}$ & $\mathrm{x}$ & $\mathrm{x}$ & $\mathrm{x}$ & & & & $\mathrm{x}$ & & $\mathrm{x}$ & $\mathrm{x}$ \\
\hline accuracy & & $\mathrm{x}$ & $\mathrm{x}$ & $\mathrm{x}$ & $\mathrm{x}$ & $\mathrm{x}$ & $\mathrm{x}$ & $\mathrm{x}$ & $\mathrm{x}$ & & & & $\mathrm{x}$ & & . & $\mathrm{x}$ \\
\hline task completion/effectiveness & & $x$ & $\mathrm{x}$ & $\mathrm{x}$ & $\mathrm{x}$ & $\mathrm{x}$ & $\mathrm{x}$ & $\mathrm{x}$ & $\mathrm{x}$ & & & & $\mathrm{x}$ & & $\mathrm{x}$ & $\mathrm{x}$ \\
\hline efficiency & & $\mathrm{x}$ & $\mathrm{x}$ & $\mathrm{x}$ & $\mathrm{x}$ & $\mathrm{x}$ & $\mathrm{x}$ & $\mathrm{x}$ & $\mathrm{x}$ & & & & $\mathrm{x}$ & & $\mathrm{x}$ & $\mathrm{x}$ \\
\hline dexterity & & $\mathrm{x}$ & $\mathrm{x}$ & $\mathrm{x}$ & $\mathrm{x}$ & $\mathrm{x}$ & $\mathrm{x}$ & $\mathrm{x}$ & $\mathrm{x}$ & & & & $\mathrm{x}$ & & $\mathrm{x}$ & $\mathrm{x}$ \\
\hline autonomy & & $\mathrm{x}$ & $\mathrm{x}$ & $\mathrm{x}$ & $\mathrm{x}$ & $\mathrm{x}$ & $\mathrm{x}$ & $\mathrm{x}$ & $\mathrm{x}$ & & & & $\mathrm{x}$ & & $\mathrm{x}$ & $\mathrm{x}$ \\
\hline stability & & $\mathrm{x}$ & $\mathrm{x}$ & $\mathrm{x}$ & $\mathrm{x}$ & $\mathrm{x}$ & $\mathrm{x}$ & $\mathrm{x}$ & $\mathrm{x}$ & & & & $\mathrm{x}$ & & $\mathrm{x}$ & $\mathrm{x}$ \\
\hline $\begin{array}{l}\text { obstacle detection and } \\
\text { avoidance }\end{array}$ & & $x$ & $\mathrm{x}$ & $\mathrm{x}$ & $x$ & $x$ & $x$ & $x$ & $x$ & & & & $x$ & & & $x$ \\
\hline $\begin{array}{l}\text { exploration of unknown } \\
\text { environments }\end{array}$ & & $x$ & $x$ & $x$ & $x$ & $x$ & $x$ & $x$ & $x$ & & & & $x$ & & & $x$ \\
\hline
\end{tabular}

\section{Performance Measurements}

This section surveys the performance measurement research for robots, mobile robots, and mobile manipulators. The literature search of robot performance measurement uncovered few published articles; mobile manipulation performance measurement, being a relatively new area of research, provided even fewer articles. Calibration, standards, and artifacts to evaluate performance are surveyed in this section.

\subsection{Calibration}

Calibration greatly improves the correspondence between the real position of the robot end effector and the position calculated from the mathematical model of the robot. Elatta et al. [109] describe parametric robot calibration as using the kinematic parameters to define models of an industrial robot. Kinematic parameters describe the relative position and orientation of links and joints of the robot while the dynamic parameters describe arm and joint masses and internal friction.

Robot calibration and metrology systems vary widely in performance, but as a general rule, they are considered to be expensive systems that are normally beyond the budget of the average company. Hidalgo and Brunn [110] performed a market survey involving some of the leading systems available and revealed that the leading performers are characteristically easy to set-up and operate, and are more economical. Nevertheless, the price range of these systems is still too high for them to be in widespread, regular use. They suggest that development of systems that combine these characteristics, but at a low-cost, would fill an important void in the automated manufacturing industry.

Greenway [111] discussed robot accuracy and repeatability and the mechanical and control aspects of robots that lead to errors occurring in static positioning and dynamic path following. He described both "string pull" devices (two examples of which are described in [112], see Fig. 5, and in [113]) and laserbased systems, that utilize laser light for both alignment and distance measurement. An example of one such laser-based system mounted a laser on a 2 axis tracking head that follows the movement of a target. Vision-based photogrammetry and videogrammetry systems, which are vision systems with a minimum of two cameras, are stationary while a fixed pattern of light sources attached to the robot moves within the field-of-view of the cameras where the location and orientation of the light sources can be calculated from the camera images.

Sensors must be calibrated both to the manipulators and to each other, since fused sensor data is often needed. Pradeep et al. [114] proposed an extendable framework that combined measurements from the robot's various sensors (proprioceptive and external) to calibrate the robot's joint offsets and external sensor locations. The framework was validated by implementing it on a commercial mobile manipulator robot, providing a significant improvement in the robot's calibration. A popular configuration widely used 


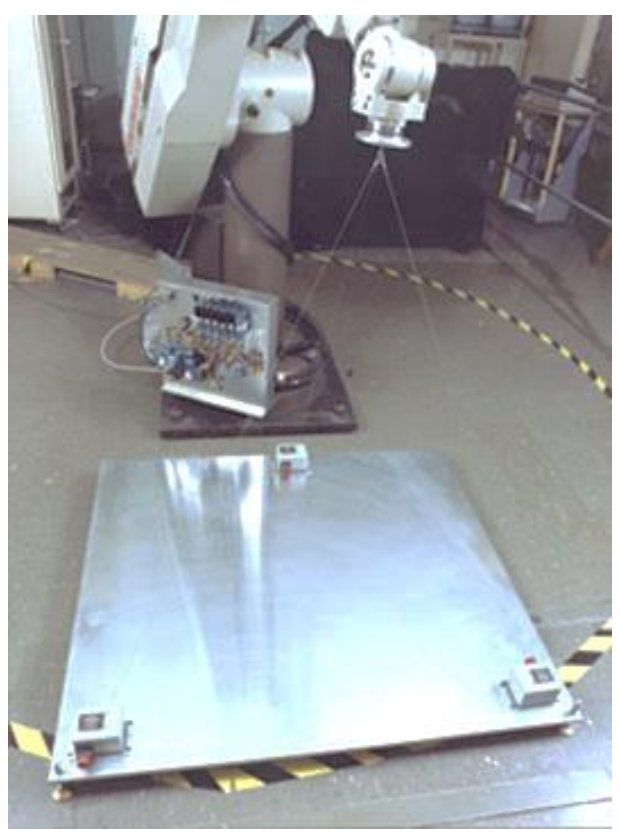

Fig. 5. NIST Robot Calibrator [112].

in a variety of robotic applications is to mount a camera on the robot manipulator hand. Before performing a measurement task using such a system, both the camera and the robot need to be calibrated. Zhuang et al. [115] discussed a procedure developed for simultaneous calibration of a robot and a monocular camera. Unlike conventional approaches based on first calibrating the camera and then calibrating the robot, the algorithm solved for the kinematic parameters of the robot and camera in one stage, thus eliminating error propagation and improving noise sensitivity. Preising and Hisa [116] demonstrated the use of a single camera 3D computer vision system as a position sensor to perform robot calibration instead of being calculated from the kinematic equations. The authors describe a vision feedback scheme they termed vision-guided robot control (VRC) which may improve the accuracy of a robot in an on-line, iterative manner. The degree of accuracy was determined by setting a tolerance level for each of the six DoF in Cartesian space. In general, a small tolerance level requires a large number of iterations in order to position the end effector, while a large tolerance level requires fewer iterations.

\subsection{Standards}

The standards landscape is represented by both safety and performance standards. For industrial robots, safety is addressed in both international (ISO 10218 parts 1, 2 [117]) and national (American National Standards Institute (ANSI)/Robotics Industry of America, RIA, 15.06 [118]) standards. AGV safety is currently defined only in national standards (e.g., ANSI/Industrial Truck Standards Development Foundation (ITSDF) B56.5 [119], and European Standards (EN BS) 1525 [120]). Industrial robot performance is also defined at both the international (ISO 9283 [121]) and national (RIA 15.05 [122]) levels. AGV performance standards are currently being developed through ASTM International Committee F45 [123].

However, these standards are not combined for mobile manipulators, and leave gaps in both safety [124] and performance. RIA began a standards development working group for mobile manipulators in 2015. At the time of writing, there are no performance standards efforts or standard test methods for mobile manipulators to compare capabilities of various manufacturers systems as they evolve. Assuming mobile manipulator safety [125] will be solved and standardized in the near-term, performance standards must follow so that potential users can compare systems against tasks. ISO 9283 and the RIA 15.05 standards depict the performance criteria and related testing methods to determine performance characteristics of 
industrial robots. They define the important performance characteristics, describe how they shall be specified, and recommend how they should be tested. The ASTM F45 committee of performance standards for automatic guided industrial vehicles includes environmental effects, docking and navigation, communication and integration, object detection and protection, and terminology areas. F45 is expected to include performance test methods for mobile manipulators where "precision" docking of the system may be expected to provide, for example, two or more orders of magnitude smaller tolerance docking than are needed for pallet acquisition for assembly or other similar tasks.

\subsection{Artifacts to Evaluate Performance}

Performance evaluation artifacts for safety of robots and mobile robots/AGVs appear in their respective safety standards. However, industrial safety system performance artifact research is non-existent for robots: a literature search provided no results and minimal results for mobile robots/AGVs [124]. Similarly, a literature search for artifacts used to measure mobile manipulator performance was virtually non-existent until recently at the U.S. National Institute of Standards and Technology (NIST) [126]. Mobile manipulator users could spend a relatively large amount of money having artifacts machined. However, advancements in additive manufacturing technologies enable rapid prototyping capabilities that significantly reduce the cost and effort necessary to produce such artifacts. Figure 6 (a) shows a graphical depiction of an artifact being developed and used at NIST for measuring performance of mobile manipulators. The artifact allows a machined surface (flat as in Fig. 6 (a) or convex/concave as in Fig. 6 (b)) with patterned holes to position reflectors and to be tilted horizontally, vertically, or at any angle beside or above the mobile manipulator. Each reflector can be positioned perpendicular to the surface or at any pitch and/or yaw angle. A laser retro-reflector is wielded by the manipulator and positioned above and in-line with each reflector to measure manipulator position accuracy (within the laser and reflector tolerance), repeatability, detection, time for detection, efficiency of motion, dexterity, and autonomy. Many of these parameters can be determined by measurement from one mobile base position. Additionally, travel distance coupled with dexterity and autonomy can also be measured using the apparatus by indexing or continuously moving the mobile base along or around the apparatus. Alternatively, the robot could wield a reflector while the apparatus houses laser retro-reflectors, resulting in a much simpler robot interface, but with greater cost from additional lasers.
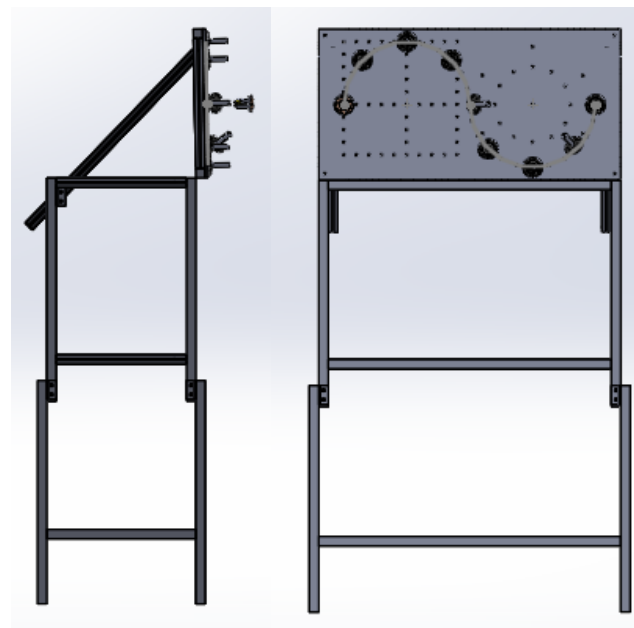

a

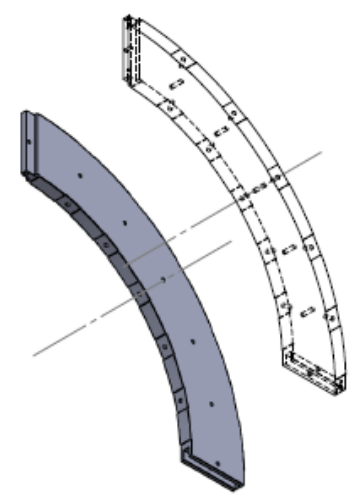

b

Fig. 6. Graphic of the NIST mobile manipulator artifact showing (a) adjustable height table with multiple geometric patterns of tapped holes and (b) flat (top/bottom), concave (inside edge), convex (outside edge) patterns of holes for mounting reflectors or laser retroreflectors and designed to mount into circular or sinusoidal shapes. 


\subsection{Robot Performance Measurement}

There have been very few published works pertaining to robot performance measurement research, especially for industrial robots. Most papers discuss the systems used to measure robot performance, instead of the test method developed, and are included in Sec. 4. Included in this section is research describing mathematical tools, measurement of a teleoperated robot, and a software framework for robot performance measurement.

Dynamic capability equations (DCE) are described by Bowling and Khatib [127] and provide a new description of robot (non-redundant manipulators with as many actuators as DoF) acceleration and force capabilities, which they called a "new tool for analyzing manipulator performance." Robot acceleration and force capabilities refer to a manipulator's ability to accelerate its end-effector and to apply forces to the environment at the end-effector. The key features in the development of these equations were that they combined the analysis of end-effector accelerations, velocities, and forces, while addressing the difference in units between translational and rotational quantities.

Although teleoperated, Hannaford et al. [128] measured performance of the flight telerobotic servicer robot in a series of generic and application-driven tasks with and without force feedback, and with control shared between teleoperation and local sensor referenced control. Measurements defining task performance included $100 \mathrm{~Hz}$ recording of six-axis force-torque information, task completion time, and visual observation of predefined task errors. Park et al. [129] evaluated methods of measuring motion characteristics (i.e., payload, repeatability, maximum speed, and position accuracy) of pose and path for a dual-arm robot and provided results.

Although not aimed at industrial robot performance measurement research, a software framework was developed in 2006 by Balakirsky et al. [130] that allowed for the realistic modeling of robots, sensors, and actuators, as well as complex, unstructured, dynamic environments. Because it was a simulation framework for these performance measurements, it could therefore be adapted to industrial applications as well. Multiple heterogeneous agents could be concurrently placed in the simulation environment thus allowing for team or group evaluations.

\subsection{Mobile Robot Performance Measurement}

Robot motion performance evaluation methods and systems remain a challenge, although substantial progress has been made in recent years. Off-line evaluation techniques are non-existent yet, evaluation is "deeply influenced by the task, the environment, and the specific representation chosen for it." Calisi and Nardi [131] concentrated on tasks that require the robot to move from one configuration to another. They describe that the task can either be an independent sub-task of a more complex plan or represent a goal by itself. After characterizing the goals and the tasks, the authors described the commonly-used problem decomposition and different kinds of modeling that could be used, from accurate metric maps to minimalistic representations.

In 2000, Baltes [132] described a benchmark suite for mobile robots that provided quantitative measurements of a mobile robot's ability to perform specific tasks. The benchmarks tested the path- and trajectory-tracking control and accuracy, the static path planning, and the dynamic path planning ability of a mobile robot. A set of metrics that provide important information about a mobile robot's performance are also presented. Baltes states that, "benchmarks could also be used as simple games which would lead to an increased opportunity for researchers to evaluate their work without having to buy expensive or special purpose equipment.” In 2014, Ceballos et al. [133] developed a mobile robot simulator for research and education that was implemented in Matlab. It modeled the robot's differential kinematics and proximity sensors. It allowed the performance assessment of navigation algorithms through various quality metrics that are useful for comparing and analyzing simulated navigation algorithms of mobile robots.

Commercially available AGV's could provide the mobility for onboard manipulators for agile manufacturing applications. To ensure that all AGV's performance characteristics are expressed in the same manner, Bostelman et al. [134] measured the performance of an AGV towards development of standard performance test methods. Measurements of the AGV navigating standard geometrically shaped 
paths (e.g., circle, square, etc.) and in various steering modes and velocities were compared to a multiple camera tracking system used as ground truth.

In 1998, Duckett and Nehmzow [135] researched self-localization in autonomous mobile robot navigation, including a general performance metric and a standard experimental procedure. The concept allows disparate localization systems to be compared on the same robot in the same environment. To demonstrate the utility of the approach taken, they tested the evidence-based localization system in six different environments, comparing its performance to that of localization using dead reckoning or currently observable landmarks.

A survey of the mobile robot competitions that fostered quite a lot of mobile robot research progress is found in [136]. A recent competition, not included in [136], is RoCKIn [137] which fostered benchmarking of robots for home and work. The survey led to the formation of the ASTM International F45 automatic guided vehicle performance standard committee. For example, one competition in the report included the US Defense Advanced Research Project Agency's Learning Applied to Ground Robots (LAGR) program, which ran from 2004 until 2008. LAGR had the goal of accelerating progress in autonomous, perceptionbased, off-road navigation in robotic unmanned ground vehicles (UGVs) using one mobile robot. In the LAGR program, mobile robot performance was measured, against other robots, by completion of a task over three consecutive attempts, learning during each run to decrease travel time to the goal. After LAGR and other surveyed competitions in [136], the Multi-Autonomous Ground Robotics International Challenge (MAGIC) 2010 [138] occurred. MAGIC invited competing teams to field squads of unmanned vehicle prototypes that autonomously coordinated, planned, and executed a series of timed tasks including classifying and responding to simulated threats and exploring/mapping diverse terrains.

\subsection{Mobile Manipulator Performance Measurement}

As there have been relatively minimal performance measures considered for robots and mobile robots with regards to industrial applications, even fewer measures have been published for mobile manipulators. As stated in the mobile robot performance measurement section, measures are deeply influenced by the task, the environment, and their specific representation. In 2007, Zadarnowska and Tchon [139] noted that there are challenges in the performance evaluation of mobile manipulators and they considered the controllability of its theoretical configuration. They stated: "It seems that this area has not been explored systematically in the literature.” For example, they reference motion generation, coordinated tasks, locomotion, and manipulation, kinematics to drive design, and trajectory planning. This survey has already discussed and referenced example planning and control where the literature only described the proposed control concepts and reported results from simulation or physical systems as either having worked or not. In other words, there was no generic measurement technique or test method to compare the various mobile manipulator control method performance nor many of the other metrics described previously.

Of the few references uncovered, the most generic physical measure used to develop controls for mobile manipulators appears in 1992 in Hootsmans et al. [140]. They emulated the performance of the mobile manipulator system by mounting a robot arm onboard a six DoF Stewart platform, parallel-link manipulator. The authors experimentally investigated the behavior of a manipulator mounted on a compliant or moving base. The system could emulate a wide variety of linear and non-linear compliant vehicles. Dynamic interaction measurements were made using a six DoF force and torque sensor mounted on top of the platform and underneath the robot arm. Using an inverse kinematic model of the platform, the required vehicle motions were translated into Stewart mechanism commands.

Simulations of stability performance measurement were conducted by Yamamoto and Yun [141] in 1994 to evaluate the performance of dynamic interactions by using a mobile manipulator model for three different trajectories: straight line with a constant velocity along a $145^{\circ}$ direction with respect to the initial heading angle, circular trajectory, and straight line with constant velocity while the arm followed an oscillatory motion along the X-axis. In 2007, Zadarnowska and Tchon [142] proposed a control theoretic methodology for defining mobile manipulator dynamic performance measures, including compliance and admittance measures. They introduced both local and global performance characteristics, including: the agility ellipsoid, the agility and mobility, the condition number, and the distortion. The authors demonstrated the measures on a mobile robot to only determine optimal motion patterns. 
And in 2010, performance measurements, although not for industrial use, were performed by Advait and Kemp [21] to evaluate completion of specific tasks for a mobile manipulator. The robot approached and grasped objects from 25 object categories that were ranked most important for robotic retrieval by motor-impaired patients. Perhaps a similar list of items could be considered for task specific, performance measurements of mobile manipulators to be used in industrial environments.

\section{Conclusion}

This survey provided an extensive literature review for mobile manipulators, including robot arm and mobile robot research since they form the basis of mobile manipulators. Beginning with a brief discussion of what types of mobile manipulators have been studied, experimental applications research is then surveyed as they have driven research in other areas. Experimental applications have been considered and will drive their rapid use in industrial settings (e.g., assembly, inspection, opening doors, material handling, etc.). After considering the desired application for mobile manipulators, development of planning and control methods has been at the forefront of mobile manipulator research. Most of the planning and control research areas for mobile manipulators have included: unified motion; trajectory planning; configuration optimization; multiple tasks; stability; obstacle avoidance; outdoor use.

The lack of metrics and quantifiable measures of performance have been considered major barriers in the development of other intelligent systems. Benchmarks are important for mobile manipulators and may even guide developments in the correct directions. As in Sec. 3, benchmarks are the framework to standards. Based on the enclosed references and on past research in robotics for industrial use, the authors therefore summarized that metrics to consider for mobile manipulators might be:

- $\quad$ time/task duration

- distance traveled

- repeatability

- accuracy

- task completion/effectiveness

- efficiency

- dexterity

- autonomy

- stability

- obstacle detection and avoidance

- exploration of unknown environments

The tools that are used to measure the performance of systems, whether machine tool or robots, were surveyed where the machine tool and part measurement systems and their methods may prove useful in, or at least contrast, measuring performance of mobile manipulators. However, motion tracking systems are the focus of the majority of literature surveyed since mobile manipulators are dynamic in nature. The survey considered various motion tracking technologies, such as: camera, multi-camera, laser, and rotating laser systems.

Lastly, performance measurement research, specific to robots, mobile robots, and mobile manipulators are surveyed, beginning with calibration. In this area, contact and non-contact calibration systems are surveyed, including string-pull, laser, vision, light emitting diode with cameras, etc. and the fused sensor algorithms. Performance, as well as safety, standards are then listed for robot and mobile robot/AGV systems where ASTM F45 will be an ideal venue for mobile manipulator performance test methods. One area that shows promise for performance measurement, including calibration, of mobile manipulators is with standard artifacts. Metrology bars or other calibrated artifacts can provide a simple, reproducible, costeffective measurement system with known accuracy and repeatability for measuring mobile manipulator performance. NIST has been developing such a system that promises a virtually unlimited number of measurement configurations for mobile manipulators.

Perhaps the least published area of mobile manipulator research is in performance measurements, including artifacts. Minimal performance measurement of robots, mobile robots, and mobile manipulators 
literature was uncovered. A few research works were published discussing software frameworks, teleoperated systems measurements, benchmarks, and localization. A survey of research that includes military competitions leading to the ASTM F45 performance standard was also included. Mobile manipulator performance measurement research was suggested as not being explored systematically. Expectedly, robot and mobile robot performance measurement research is more prevalent than for mobile manipulators since this area is relatively new. However, robot and mobile robot measurement provides excellent basis for mobile manipulator measurement methods and is included in the survey. The survey is useful to practitioners as a sound basis for mobile manipulator implementation for planning, control, stability, indoor and outdoor use, etc. followed by a comparison of technologies and research results that support mobile manipulator application. Since artifacts provide such a simple, yet useful method for measuring mobile manipulator performance, it is in this area of mobile manipulator performance measurement and test method development that are suggestions or challenges to the research community.

The authors recommend the following research be considered for measuring the performance of mobile manipulator systems:

- Exhaust the usefulness of measurement systems that are unconstrained to mobile manipulator testing in a variety of applications (e.g., navigation, assembly, material handling),

- Develop performance standards based on performance research for mobile manipulators when used in a variety of applications and environments.

\section{Acknowledgments}

The authors thank Sebti Foufou, Qatar University, for his guidance in this research. Also, the authors thank the authors of research papers referenced in this body of work. Their enormous efforts combine into the evolution of complex, yet highly useful mobile manipulator systems where performance measurements will soon provide the repeatable methods to aid system design and comparison to applications.

\section{References}

[1] Shneier M, Bostelman R (2014) Literature Review of Mobile Robots for Manufacturing (National Institute of Standards and Technology, Gaithersburg, MD), NIST Internal Report 8022. http://dx.doi.org/10.6028/NIST.IR.8022

[2] Yaskawa Motoman (2013) MH80 robot unloading trucks - from Wynright Corporation. http://www.youtube.com/watch?v=8wngL0BnF_4

[3] Guizzo E (2011) Meka Robotics Announces Mobile Manipulator With Kinect and ROS. http://spectrum.ieee.org/automaton/robotics/humanoids/meka-robotics-announces-mobile-manipulator-with-kinect-and-ros

[4] Green T (2014) KUKA Falls First, Buys Swisslog for \$335M. Who’s Next? Robotics Business Review.

[5] Saggini E, Zereik E, Bibuli M, Bruzzone G, Caccia M, Riccomagno E (2014) Performance Indices for Evaluation and Comparison of Unmanned Marine Vehicles' Guidance Systems. 19th World Congress The International Federation of Automatic Control (Cape Town, South Africa).

[6] Lizarralde F, Wen JT, Hsu L (1995) Quaternion-based coordinated control of a subsea mobile manipulator with only position measurements. In Proceedings of the 34th IEEE Conference on Decision and Control (IEEE), vol 4, pp 3996-4001.

[7] Van Henten EJ, Van Tuijl BAJ, Hemming J, Kornet JG, Bontsema J, Van Os EA (2003) Field Test of an Autonomous Cucumber Picking Robot. Biosys Eng 86(3):305-313. http://dx.doi.org/10.1016/j.biosystemseng.2003.08.002

[8] Hannaford B, Wood L, McAffee DA, Zak H (1991) Performance evaluation of a six-axis generalized force-reflecting teleoperator. IEEE Transactions on Systems, Man, and Cybernetics 21(3):620-633. http://dx.doi.org/10.1109/21.97455

[9] Miksch W, Schroeder D (1992) Performance-Functional Based Controller-Design for a Mobile Manipulator. In Proceedings of the IEEE International Conference on Robotics and Automation, pp 227-232. http://dx.doi.org/10.1109/ROBOT.1992.220258

[10] Brock O, Grupen R (2005) Final report for the NSF/NASA Workshop on Autonomous Mobile Manipulation (AMM). http://www.robotics.cs.umass.edu/amm/results.html

[11] Bøgh S, Hvilshøj M, Kristiansen M, Madsen O (2011) Autonomous Industrial Mobile Manipulation (AIMM): From Research to Industry. Automate (Chicago, IL).

[12] Hamner B, Koterba S, Shi J, Simmons R, Singh S (2010) An autonomous mobile manipulator for assembly tasks. Auton Robot 28(1):131-149. http://dx.doi.org/10.1007/s10514-009-9142-y

[13] Hvilshoj M, Bogh S (2011) “Little Helper” - An Autonomous Industrial Mobile Manipulator Concept. Int J Adv Robot Syst 8(2):80-90. http://dx.doi.org/10.5772/10579

[14] Caroe C, Hvilshoj K, Schou C (2012) Rotor Shaft Assembly using the KUKA LWR. http://www.youtube.com

[15] Flannigan C (2012) Mobile Manipulation Robotics \& Automation Engineering, Southwest Research Institute. http://www.swri.org/4org/d10/msd/automation/mobile-manipulator.htm 
[16] Yingshu C, Libing L, Minglu Z, Hui R (2006) Study on Coordinated Control and Hardware System of a Mobile Manipulator. 6th World Congress on Intelligent Control and Automation, pp 9037-9041. http://dx.doi.org/10.1109/WCICA.2006.1713748

[17] Peterson L, Austin D, Kragic D (2000) High-level control of a mobile manipulator for door opening. In Proceedings of the 2000 IEEE/RSJ International Conference on Intelligent Robots and Systems, vol 2333, pp 2333-2338. http://dx.doi.org/10.1109/IROS.2000.895316

[18] Nagatani K, Yuta S (1996) Designing strategy and implementation of mobile manipulator control system for opening door. In Proceedings of the 1996 IEEE International Conference on Robotics and Automation, vol 2823, pp 2828-2834. http://dx.doi.org/10.1109/ROBOT.1996.506591

[19] Chitta S, Cohen B, Likhachev M (2010) Planning for autonomous door opening with a mobile manipulator. In Proceedings of the 2010 IEEE International Conference on Robotics and Automation, pp 1799-1806. http://dx.doi.org/10.1109/ROBOT.2010.5509475

[20] Pin FG, Culioli JC (1992) Optimal Positioning of Combined Mobile Platform-Manipulator Systems for Material Handling Tasks. J Intell Robot Syst 6(2-3):165-182. http://dx.doi.org/10.1007/BF00248014

[21] Jain A, Kemp CC (2010) EL-E: an assistive mobile manipulator that autonomously fetches objects from flat surfaces. Auton Robot 28(1):45-64. http://dx.doi.org/10.1007/s10514-009-9148-5

[22] Agah A, Tanie K (1997) Human interaction with a service robot: mobile-manipulator handing over an object to a human. In Proceedings of the 1997 IEEE International Conference on Robotics and Automation, vol 571, pp 575-580. http://dx.doi.org/10.1109/ROBOT.1997.620098

[23] Tomizawa T, Ohya A, Yuta S (2003) Remote book browsing system using a mobile manipulator. In Proceedings of the ICRA '03 IEEE International Conference on Robotics and Automation, vol 251, pp 256-261. http://dx.doi.org/10.1109/ROBOT.2003.1241605

[24] Bort L, Pobil APd (2000) Using speech to guide a mobile robot manipulator.In Proceedings of the 2000 IEEE International Conference on Systems, Man, and Cybernetics, vol 2354, pp 2356-2361. http://dx.doi.org/10.1109/ICSMC.2000.884343

[25] Holmberg R, Khatib O (2000) Development and control of a holonomic mobile robot for mobile manipulation tasks. Int J Robot Res 19(11):1066-1074. http://dx.doi.org/10.1177/02783640022067977

[26] Khatib O, Yokoi K, Chang K, Ruspini D, Holmberg R, Casal A (1996) Vehicle/arm coordination and multiple mobile manipulator decentralized cooperation. In Proceedings of the 1996 IEEE/RSJ International Conference on Intelligent Robots and Systems, vol 542, pp 546-553. http://dx.doi.org/10.1109/IROS.1996.570849

[27] Khatib O, Yokoi K, Chang K, Ruspini D, Holmberg R, Casal A, Baader A (1995) Force Strategies for Cooperative Tasks in Multiple Mobile Manipulation Systems. Internal Symposium of Robotics Research (Munich, Germany).

[28] Osumi H, Terasawa M, Nojiri H (1998) Cooperative control of multiple mobile manipulators on uneven ground. In Proceedings of the 1998 IEEE International Conference on Robotics and Automation, vol 3194, pp 3198-3203. http://dx.doi.org/10.1109/ROBOT.1998.680917

[29] Sugar T, Kumar V (1998) Decentralized control of cooperating mobile manipulators. In Proceedings of the 1998 IEEE International Conference on Robotics and Automation, vol 2914, pp 2916-2921. http://dx.doi.org/10.1109/ROBOT.1998.680672

[30] Stroupe A, Huntsberger T, Okon A, Aghazarian H, Robinson M (2005) Behavior-based multi-robot collaboration for autonomous construction tasks. 2005 IEEE/RSJ International Conference on Intelligent Robots and Systems, pp 1495-1500. http://dx.doi.org/10.1109/IROS.2005.1545269

[31] Madsen O, Bogh S, Schou C, Andersen RS, Damgaard JS, Pedersen MR, Kruger V (2015) Integration of mobile manipulators in an industrial production. Ind Robot 42(1):11-18. http://dx.doi.org/10.1108/Ir-09-2014-0390

[32] Gardner JF, Velinsky SA (2000) Kinematics of mobile manipulators and implications for design. J Robotic Syst 17(6):309-320 http://dx.doi.org/10.1002/(SICI)1097-4563(200006)17:6<309::AID-ROB2>3.0.CO;2-9

[33] Papadopoulos E, Poulakakis J (2000) Planning and model-based control for mobile manipulators. In Proceedings of the 2000 IEEE/RSJ International Conference on Intelligent Robots and Systems, vol 1813, pp 1810-1815. http://dx.doi.org/10.1109/IROS.2000.895234

[34] Jinhyun K, Wan Kyun C, Youngil Y, Lee BH (2002) Real-time ZMP compensation method using motion for mobile manipulators. In Proceedings of the 2002 IEEE International Conference on Robotics and Automation, vol 1962, pp 19671972. http://dx.doi.org/10.1109/ROBOT.2002.1014829

[35] Nagatani K, Hirayama T, Gofuku A, Tanaka Y (2002) Motion planning for mobile manipulator with keeping manipulability. In Proceedings of the 2002 IEEE/RSJ International Conference on Intelligent Robots and Systems, vol 1662, pp 1663-1668. http://dx.doi.org/10.1109/IRDS.2002.1043994

[36] Katz D, Horrell E, Yang Y, Burns B, Buckley T, Grishkan A, Zhylkovskyy V, Brock O, Learned-Miller EG (2006) “The UMass Mobile Manipulator UMan: An Experimental Platform for Autonomous Mobile Manipulation” The University of Pennsylvania. http://works.bepress.com/erik_learned_miller/37/

[37] Mailah M, E. Pitowarno E, Jamaluddin H (2005) Robust motion control for mobile manipulator using resolved acceleration and proportional-integral active force control. Int J Adv Robot Syst 2(2):125-134. http://dx.doi.org/10.5772/5794

[38] Chen MW, Zalzala AMS (1997) Dynamic modelling and genetic-based trajectory generation for non-holonomic mobile manipulators. Control Eng Pract 5(1):39-48. http://dx.doi.org/10.1016/S0967-0661(96)00205-5

[39] Tanner HG, Kyriakopoulos KJ (2001) Mobile manipulator modeling with Kane’s approach. Robotica 19:675-690.

[40] Mohri A, Furuno S, Yamamoto M (2001) Trajectory planning of mobile manipulator with end-effector's specified path. In Proceedings of the 2001 IEEE/RSJ International Conference on Intelligent Robots and Systems, vol 2264, pp 2264-2269. http://dx.doi.org/10.1109/IROS.2001.976407

[41] Dong Hun S, Hamner BS, Singh S, Myung H (2003) Motion planning for a mobile manipulator with imprecise locomotion. In Proceedings of the 2003 IEEE/RSJ International Conference on Intelligent Robots and Systems, vol 841, pp 847-853. http://dx.doi.org/10.1109/IROS.2003.1250735 
[42] Tang CP, Miller PT, Krovi VN, Ryu JC, Agrawal SK (2011) Differential-Flatness-Based Planning and Control of a Wheeled Mobile Manipulator - Theory and Experiment. IEEE/ASME Transactions on Mechatronics 16(4):768-773. http://dx.doi.org/10.1109/TMECH.2010.2066282

[43] Yamamoto Y, Yun X (1992) Coordinating locomotion and manipulation of a mobile manipulator. Proceedings of the 31st IEEE Conference on Decision and Control, vol 264,3 pp 2643-2648. http://dx.doi.org/10.1109/CDC.1992.371337

[44] Chen M, Zalzala AMS (1997) A genetic approach to motion planning of redundant mobile manipulator systems considering safety and configuration. J Robotic Syst 14(7):529-544. http://dx.doi.org/10.1002/(SICI)1097-4563(199707)14:7<529::AIDROB2>3.0.CO;2-P

[45] Pin FG, Culioli JC, Reister DB (1994) Using minimax approaches to plan optimal task commutation configurations for combined mobile platform-manipulator systems. IEEE Transactions on Robotics and Automation 10(1):44-54. http://dx.doi.org/10.1109/70.285584

[46] Lee JK, Cho HS (1997) Mobile manipulator motion planning for multiple tasks using global optimization approach. J Intell Robot Syst 18(2):169-190. http://dx.doi.org/10.1023/A:1007939823675

[47] Dubowsky S, Vance EE (1989) Planning mobile manipulator motions considering vehicle dynamic stability constraints. In Proceedings of the 1989 IEEE International Conference on Robotics and Automation, vol 1273, pp 1271-1276. http://dx.doi.org/10.1109/ROBOT.1989.100155

[48] Dubowski S, Gu P-Y, Deck JF (1991) The dynamic analysis of flexibility in mobile robotic manipulator systems. Eighth World Congress on the Theory of Machines and Mechanisms, (Prague, Czechoslovakia).

[49] Huang Q, Sugano S, Kato I (1994) Stability control for a mobile manipulator using a potential method. In Proceedings of the IEEE/RSJ/GI International Conference on Intelligent Robots and Systems, vol 832, pp 839-846. http://dx.doi.org/10.1109/IROS.1994.407542

[50] Qiang H, Sugano S (1995) Manipulator motion planning for stabilizing a mobile-manipulator. In Proceedings of the 1995 IEEE/RS International Conference on Intelligent Robots and Systems, vol 463, pp 467-472. http://dx.doi.org/10.1109/IROS.1995.525926

[51] Huang Q, Sugano S (1996) Motion planning of stabilization and cooperation of a mobile manipulator-vehicle motion planning of a mobile manipulator. In Proceedings of the 1996 IEEE/RSJ International Conference on Intelligent Robots and Systems, vol 562, pp 568-575. http://dx.doi.org/10.1109/IROS.1996.570852

[52] Sugano S, Huang Q, Kato I (1993) Stability criteria in controlling mobile robotic systems. In Proceedings of the 1993 IEEE/RSJ International Conference on Intelligent Robots and Systems, vol 832, pp 832-838. http://dx.doi.org/10.1109/IROS.1993.583186

[53] Qiang H, Sugano S, Tanie K (1998) Motion planning for a mobile manipulator considering stability and task constraints. Proceedings of the 1998 IEEE International Conference on Robotics and Automation, vol 219,3 pp 2192-2198. http://dx.doi.org/10.1109/ROBOT.1998.680649

[54] Huang Q, Tanie K, Sugano S (1999) Stability compensation of a mobile manipulator by manipulator motion: feasibility and planning. Adv Robotics 13(1):25-40.

[55] Huang Q, Tanie K, Sugano S (2000) Coordinated motion planning for a mobile manipulator considering stability and manipulation. Int J Robot Res 19(8):732-742. http://dx.doi.org/10.1177/02783640022067139

[56] Inoue F, Muralami T, Ihnishi K (2001) A motion control of mobile manipulator with external force. IEEE/ASME Transactions on Mechatronics 6(2):137-142. http://dx.doi.org/10.1109/3516.928727

[57] Furuno S, Yamamoto M, Mohri A (2003) Trajectory planning of mobile manipulator with stability considerations. In Proceedings of the ICRA 2003 IEEE International Conference on Robotics and Automation, vol 3403, pp 3403-3408. http://dx.doi.org/10.1109/ROBOT.2003.1242116

[58] Shibata T, Murakami T (2007) A Null Space Control of Two Wheels Driven Mobile Manipulator Using Passivity Theory. IEEJ Transactions on Industry Applications 127(11):1109-1116. http://dx.doi.org/10.1541/ieejias.127.1109

[59] Papadopoulos EG, Rey DA (1996) A new measure of tipover stability margin for mobile manipulators. In Proceedings of the 1996 IEEE International Conference on, vol 4, pp 31S11-3116. http://dx.doi.org/10.1109/ROBOT.1996.509185

[60] Papadopoulos E, Rey DA (2000) The force-angle measure of tipover stability margin for mobile manipulators. Veh Syst Dyn 33(1):29-48. http://dx.doi.org/10.1076/0042-3114(200001)33:1;1-5;Ft029

[61] Thibodeau BJ, Deegan P, Grupen R (2006) Static analysis of contact forces with a mobile manipulator. In Proceedings of the 2006 IEEE International Conference on Robotics and Automation, pp 4007-4012. http://dx.doi.org/10.1109/ROBOT.2006.1642317

[62] Khatib O (1986) Real-Time Obstacle Avoidance for Manipulators and Mobile Robots. Int J Robot Res 5(1):90-98. http://dx.doi.org/10.1177/027836498600500106

[63] Yamamoto Y, Xiaoping Y (1995) Coordinated obstacle avoidance of a mobile manipulator. Proceedings of the 1995 IEEE International Conference on Robotics and Automation, vol 2253, pp 2255-2260. http://dx.doi.org/10.1109/ROBOT.1995.525597

[64] Yamamoto Y, Fukuda S (2002) Trajectory planning of multiple mobile manipulators with collision avoidance capability. Robotics and Automation, 2002 Proceedings of the ICRA 2002 IEEE International Conference on, vol 3564, pp 3565-3570. http://dx.doi.org/10.1109/ROBOT.2002.1014262

[65] Nowak W, Zakharov A, Blumenthal S, and Prassler E (2010) Benchmarks for mobile manipulation and robust obstacle avoidance and navigation (Best Practices in Robotics [BRICS]), BRICs Deliverable D3.1.

[66] Hootsmans NAM, Dubowsky S, Mo PZ (1992) The experimental performance of a mobile manipulator control algorithm. In Proceedings of the 1992 IEEE International Conference on Robotics and Automation, vol 1943, pp 1948-1954. http://dx.doi.org/10.1109/ROBOT.1992.219944

[67] Najjaran H, Goldenberg A (2007) Real-time motion planning of an autonomous mobile manipulator using a fuzzy adaptive Kalman filter. Robot Auton Syst 55(2):96-106. http://dx.doi.org/10.1002/j.robot.2006.07.002 
[68] Wells P, Deguire D (2005) TALON: a universal unmanned ground vehicle platform, enabling the mission to be the focus. In Proceedings of the SPIE - International Society for Optics and Photonics, vol 5804, pp 747-757. http://dx.doi.org/10.1117/12.602887

[69] Yang Y and O. Brock (2006) Elastic roadmaps: Globally task-consistent motion for autonomous mobile manipulation. In Proceedings of Robotics: Science and Systems (RSS) (Philadelphia, USA).

[70] Graettinger TJ, Krogh BH (1988) The acceleration radius: a global performance measure for robotic manipulators. IEEE Journal on Robotics and Automation 4(1):60-69. http://dx.doi.org/10.1109/56.772

[71] Chung JH, Velinsky SA (1998) Modeling and control of a mobile manipulator. Robotica 16:607-613. http://dx.doi.org/10.1017/S0263574798000873

[72] Watanabe K, Sato K, Izumi K, Kunitake Y (2000) Analysis and control for an omnidirectional mobile manipulator. J Intell Robot Syst 27(1-2):3-20. http://dx.doi.org/10.1023/A:1008145910986

[73] Mbede JB, Ele P, Mveh-Abia CM, Toure Y, Graefe V, Ma SG (2005) Intelligent mobile manipulator navigation using adaptive neuro-fuzzy systems. Inform Sciences 171(4):447-474. http://dx.doi.org/10.1016/j.ins.2004.09.014

[74] Korayem MH, Ghariblu H (2004) Analysis of wheeled mobile flexible manipulator dynamic motions with maximum load carrying capacities. Robot Auton Syst 48(2-3):63-76. http://dx.doi.org/10.1016/j.robot.2004.07.010

[75] Albus JS (2002) Metrics and performance measures for intelligent unmanned ground vehicles. In Proceedings of the Performance Metrics for Intelligent Systems (PerMIS) Workshop (National Institute of Standards and Technology, Gaithersburg, MD).

[76] Hägele M, Pfeiffer K (2007) Report on State of the Art on Benchmarks for Mobile Manipulation and Service Robots (Fraunhofer IPA), RoSta Deliverable D 4.1.

[77] Edan Y, Friedman L, Mehrez A, Slutski L (1998) A three-dimensional statistical framework for performance measurement of robotic systems. Robot Cim-Int Manuf 14(4):307-315. http://dx.doi.org/10.1016/S0736-5845(98)00006-4

[78] Wong SC, Middleton L, MacDonald BA, Auckland N (2002) Performance metrics for robot coverage tasks. In Proceedings of Australasian Conference on Robotics and Automation, vol 27, pp 29.

[79] Sprunk C, Röwekämper J, Parent G, Spinello L, Tipaldi GD, Burgard W, Jalobeanu M (2014) An Experimental Protocol for Benchmarking Robotic Indoor Navigation. In Proceedings of the International Symposium on Experimental Robotics (ISER).

[80] Ioan S, Kavraki LE (2010) On the implementation of single-query sampling-based motion planners. In Proceedings of the 2010 IEEE International Conference on Robotics and Automation, pp 2005-2011. http://dx.doi.org/10.1109/ROBOT.2010.5509172

[81] Bonsignorio F, Hallam J, del Pobil A (2009) Defining the requisites of a replicable robotics experiment. RSS2009 Workshop on Good Experimental Methodologies in Robotics (Seattle, WA).

[82] Jacoff A, Messina E, Weiss BA, Tadokoro S, Nakagawa Y (2003) Test arenas and performance metrics for urban search and rescue robots. In Proceedings of the 2003 IEEE/RSJ International Conference on Intelligent Robots and Systems, vol 3393, pp 3396-3403. http://dx.doi.org/10.1109/IROS.2003.1249681

[83] Lampe A, Chatila R (2006) Performance measure for the evaluation of mobile robot autonomy. In Proceedings of the 2006 IEEE International Conference on Robotics and Automation, pp 4057-4062. http://dx.doi.org/10.1109/ROBOT.2006.1642325

[84] Steinfeld A, Fong T, Kaber D, Lewis M, Scholtz J, Schultz A, Goodrich M (2006) Common metrics for human-robot interaction. In Proceedings of the 1st ACM SIGCHI/SIGART Conference on Human-robot Interaction, pp 33-40.

[85] Canfield SL, Langley D, Shibakov A (2013) Developing Metrics for Comparison of Mobile Robots Performing Welding Tasks. In Proceedings of the ASME 2013 International Design Engineering Technical Conferences and Computers and Information in Engineering Conference (Portland, Oregon).

[86] Eastman R, Hong T, Shi J, Hanning T, Muralikrishnan B, Young S, Chang T (2010) Performance Evaluation and Metrics for Perception in Intelligent Manufacturing. Performance Evaluation and Benchmarking of Intelligent Systems, eds Madhavan R, Tunstel E, Messina E (Springer US), pp 269-310.

[87] Ilnicki R, Zadarnowska K (2009) Efficiency of double nonholonomic mobile manipulators. In Proceedings of the 14th International Conference on Methods and Models in Automation and Robotics, vol 14, pp 641-646. http://dx.doi.org/10.3182/20090819-3-PL-3002.00111

[88] Shiakolas PS, Conrad KL, Yih TC (2002) On the Accuracy, Repeatability, and Degree of Influence of Kinematics Parameters for Industrial Robots. International Journal of Modelling and Simulation 22(4):245-254. http://dx.doi.org/10.1080/02286203.2002.11442246

[89] Choi JP, Min BK, Lee SJ (2004) Reduction of machining errors of a three-axis machine tool by on-machine measurement and error compensation system. J Mater Process Tech 155:2056-2064. http://dx.doi.org/10.1016/j.jmatprotec.2004.04.402

[90] Liu H, Darabi H, Banerjee P, Liu J (2007) Survey of Wireless Indoor Positioning Techniques and Systems. IEEE Transactions on Systems, Man, and Cybernetics, Part C (Applications and Reviews) 37(6):1067-1080. http://dx.doi.org/10.1109/TSMCC.2007.905750

[91] Angeles J, Park FC (2008) Performance Evaluation and Design Criteria. Springer Handbook of Robotics, eds Siciliano B, Khatib O (Springer Berlin Heidelberg, Berlin, Heidelberg), pp 229-244.

[92] ASTM E2919-14 (2014) Standard Test Method for Evaluating the Performance of Systems that Measure Static, Six Degrees of Freedom (6DOF), Pose, ASTM International, West Conshohocken, PA. http://dx.doi.org/10.1520/E2919-14

[93] ASTM WK49831 (2015) Standard Test Method for Evaluating the Performance of Systems that Measure Static and Dynamic, Six Degrees of Freedom (6DOF), Pose Working Document.

[94] Bostelman R, Falco J, Hong T (2015) Performance Measurements of Motion Capture Systems used for AGV and Robot Arm Evaluation. 6th Computer Vision in Vehicle Technology Workshop (CVVT) at CVPR 2015 (Boston, MA).

[95] University of Minnesota (2015) Virtual Reality Design Lab. http://vr.design.umn.edu/technology/

[96] Dainis A, Juberts M (1985) Accurate remote measurement of robot trajectory motion. In Proceedings of the 1985 IEEE International Conference on Robotics and Automation, pp 92-99. http://dx.doi.org/10.1109/ROBOT.1985.1087317

[97] Murray D, Basu A (1994) Motion tracking with an active camera. IEEE Transactions on Pattern Analysis and Machine Intelligence 16(5):449-459. http://dx.doi.org/10.1109/34.291452 
[98] Foxlin E (2002) Motion tracking requirements and technologies. Handbook of virtual environment technology 8:163-210.

[99] Foxlin EM (2001, 2008) Motion tracking system. (U.S. Patent 6,176,837, US Patent 7,395,181)

[100] Lau K, Hocken R, Haynes L (1985) Robot performance measurements using automatic laser tracking techniques. Robot CimInt Manuf 2(3):227-236. http://dx.doi.org/10.1016/0736-5845(85)90110-3

[101] Prenninger JP (1993) Contactless position and orientation measurement of robot end-effectors. In Proceedings of the 1993 IEEE International Conference on Robotics and Automation, vol 181, pp 180-185. http://dx.doi.org/10.1109/ROBOT.1993.291980

[102] Estler WT, Edmundson KL, Peggs GN, Parker DH (2002) Large-Scale Metrology - An Update. CIRP Annals - Manufacturing Technology 51(2):587-609. http://dx.doi.org/10.1016/S0007-8506(07)61702-8

[103] Qiao H (2015) Advanced Sensing, Automation, and Manufacturing Information Integration, Automated Precision, Inc. Lecture at the National Institute of Standards and Technology (Gaithersburg, MD).

[104] Mayer JRR, Parker GA (1994) A portable instrument for 3-D dynamic robot measurements using triangulation and laser tracking. IEEE Transactions on Robotics and Automation 10(4):504-516. http://dx.doi.org/10.1109/70.313100

[105] Slamani M, Nubiola A, Bonev I (2012) Assessment of the positioning performance of an industrial robot. Ind Robot 39(1):5768. http://dx.doi.org/10.1108/01439911211192501

[106] Tso SK, Wang TW, Xu WL, Sun ZQ (2003) Vibration control for a flexible-link robot arm with deflection feedback. Int J Nonlin Mech 38(1):51-62. http://dx.doi.org/10.1016/S0020-7462(01)00040-3

[107] Schmitt R, Nisch S, Schönberg A, Demeester F, Renders S (2010) Performance evaluation of iGPS for industrial applications. In Proceedings of the 2010 International Conference on Indoor Positioning and Indoor Navigation, pp 1-8. http://dx.doi.org/10.1109/IPIN.2010.5647630

[108] Nikon Metrology (2014) iGPS System. http://www.nikonmetrology.com/en_US/Products/Large-VolumeApplications/iGPS/iGPS/(key_features)

[109] Elatta A, Gen LP, Zhi FL, Daoyuan Y, Fei L (2004) An overview of robot calibration. Information Technology Journal 3(1):74-78. http://dx.doi.org/10.3923/itj.2004.74.78

[110] Hidalgo F, Brunn P (1998) Robot metrology and calibration systems - a market review. Industrial Robot 25(1):42-47. http://dx.doi.org/10.1108/01439919810196955

[111] Greenway B (2000) Robot accuracy. Industrial Robot: An International Journal 27(4):257-265. http://dx.doi.org/10.1108/01439910010372136

[112] NIST Robot Calibrator (2011). http://www.nist.gov/el/isd/robot-calibrator.cfm

[113] Driels MR, Swayze WE (1994) Automated partial pose measurement system for manipulator calibration experiments. IEEE Transactions on Robotics and Automation 10(4):430-440. http://dx.doi.org/10.1109/70.313094

[114] Pradeep V, Konolige K, Berger E (2010) Calibrating a multi-arm multi-sensor robot: A Bundle Adjustment Approach. International Symposium on Experimental Robotics (ISER) (New Delhi, India).

[115] Hanqi Z, Kuanchih W, Roth ZS (1995) Simultaneous calibration of a robot and a hand-mounted camera. IEEE Transactions on Robotics and Automation 11(5):649-660. http://dx.doi.org/10.1109/70.466601

[116] Preising B, Hsia TC (1995) Robot Performance-Measurement and Calibration Using a 3d Computer Vision System. Robotica 13(4):327-337. http://dx.doi.org/10.1017/S0263574700018762

[117] International Organization of Standardization, ISO 10218-1. Robots and robotic devices - Safety requirements - Part 1 and Robots, ISO 10218-2. Robots and robotic devices - Safety requirements - Part 2: Industrial robot sys-tems and integration, 2011.

[118] ANSI/RIA R15.06 (2012) American National Standard for Industrial Robots and Robot Systems- Safety Requirements.

[119] Industrial Truck Standards Development Foundation (2014) ANSI/ITSDF B56.5-2012, Safety Standard for Driverless, Automatic Guided Industrial Vehicles and Automated Functions of Manned Industrial Vehicles.

[120] European Standards (1998) EN BS 1525: Safety of industrial trucks. Driverless trucks and their systems.

[121] ISO 9283 (1998) Manipulating industrial robotos - Performance criteria and related test methods.

[122] ANSI/RIA R15.05 (1999) Evaluation of Point-to-Point and Static Performance Characteristics of Industrial Robots and Robot Systems.

[123] ASTM F45 (2015) 2014 Driverless Automatic Industrial Vehicles. https://www.astm.org/COMMITTEE/F45.htm

[124] Marvel J, Bostelman R (2013) Towards mobile manipulator safety standards. In Proceedings of the 2013 IEEE International Symposium on Robotic and Sensors Environments, pp 31-36. http://dx.doi.org/10.1109/ROSE.2013.6698414

[125] Bostelman R, Marvel J (2014) Control fusion for safe multi-robot coordination. In Proceedings of the SPIE - International Society for Optics and Photonics, vol 9121. http://dx.doi.org/10.1117/12.2051410

[126] Bostelman R, Hong T, Marvel J (2015) Performance measurement of mobile manipulators. In Proceedings of the SPIE International Society for Optics and Photonics, vol 9498. http://dx.doi.org/10.1117/12.2177344

[127] Bowling A, Khatib O (2005) The dynamic capability equations: a new tool for analyzing robotic manipulator performance. IEEE Transactions on Robotics 21(1):115-123. http://dx.doi.org/10.1109/TRO.2004.837243

[128] Hannaford B, Wood L, McAffee DA, Zak H (1991) Performance evaluation of a six-axis generalized force-reflecting teleoperator. IEEE Transactions on Systems, Man, and Cybernetics 21(3):620-633. http://dx.doi.org/10.1109/21.97455

[129] Park KT, Park CH, Shin YJ (2008) Performance Evaluation of Industrial Dual-Arm Robot. In Proceedings of the 2008 International Conference on Smart Manufacturing Application, pp 437-440. http://dx.doi.org/10.1109/ICSMA.2008.4505596

[130] Balakirsky S, Scrapper C, Carpin S, Lewis M (2006) USARSim: providing a framework for multi-robot performance evaluation. In Proceedings of PerMIS, vol. 2006.

[131] Calisi D, Nardi D (2009) Performance evaluation of pure-motion tasks for mobile robots with respect to world models. Auton Robot 27(4):465-481. http://dx.doi.org/10.1007/s10514-009-9150-y

[132] Baltes J (2000) A Benchmark Suite for Mobile Robots. In Proceedings of the 2000 IEEE/RSJ International Conference on Intelligent Robots and Systems, vol 1102, pp 1101-1106. http://dx.doi.org/10.1109/IROS.2000.893166 
[133] Munoz Ceballos N, Valencia J, Alvarez Giraldo A (2014) Simulation and Assessment Educational Framework for Mobile Robot Algorithms. Journal of Automation, Mobile Robotics \& Intelligent Systems 8(1):53-59. http://dx.doi.org/10.14313/JAMRIS_1-2014/7

[134] Bostelman R, Hong T, Cheok G (2015) Navigation Performance Evaluation for Automated Guided Vehicles. 7th Annual IEEE International Conference on Technologies for Practical Robot Applications (TePRA) (Boston, MA).

[135] Duckett T, Nehmzow U (1998) Mobile robot self-localisation and measurement of performance in middle-scale environments. Robot Auton Syst 24(1-2):57-69. http://dx.doi.org/10.1016/S0921-8890(98)00022-0

[136] Bostelman R, Messina E (2016) Towards Development of an Automated Guided Vehicle Intelligence Level Performance Standard. utonomous Industrial Vehicles: From the Laboratory to the Factory Floor, eds Bostelman R, Messina E (ASTM International, West Conshohocken, PA). http://dx.doi.org/10.1520/STP159420150054

[137] RoCKIn project (2013) RoCKIn Competition (European Union contract no. FP7-ICT-601012). http://rockinrobotchallenge.eu/index.php

[138] Robotic Industries Association (2009) Robots for the Battlefield: 12 Finalists in Worldwide Competition Go for the Prize in 2010. http://www.robotics.org/

[139] Zadarnowska K, Tchoń K (2007) Kinematic Motion Patterns of Mobile Manipulators. Robot Motion and Control 2007, ed Kozłowski K (Springer London, London), pp 209-217.

[140] Hootsmans NAM, Dubowsky S, Mo PZ (1992) The experimental performance of a mobile manipulator control algorithm. In Proceedings of the 1992 IEEE International Conference on Robotics and Automation, vol 1943, pp 1948-1954. http://dx.doi.org/10.1109/ROBOT.1992.219944

[141] Yamamoto Y, Xiaoping Y (1994) Modeling and compensation of the dynamic interaction of a mobile manipulator. In Proceedings of the 1994 IEEE International Conference on Robotics and Automation, vol 2183, pp 2187-2192. http://dx.doi.org/10.1109/ROBOT.1994.350960

[142] Zadarnowska K, Tchon K (2007) A control theory framework for performance evaluation of mobile manipulators. Robotica 25:703-715. http://dx.doi.org/10.1017/S0263574707003803

About the authors: Roger Bostelman is an electrical engineer in the Manipulation and Mobility Group of the Intelligent Systems Division of the Engineering Laboratory at NIST. He carries out research in robotics safety and performance, specifically in mobile manipulation and advanced mobility to meet demands of the manufacturing industry.

Tsai Hong is a computer scientist in the Cognition and Collaboration System Group of the intelligent System Division of the Engineering Laboratory at NIST. She carries out research in computer vision, machine learning, sensor calibration and registration, object recognition and pose determination, and performance measurement.

Jeremy Marvel is a computer scientist in the Manipulation and Mobility Group of the Intelligent Systems Division of the Engineering Laboratory at NIST. He carries out research in collaborative robotics and artificial intelligence to enable the advancement of next-generation robotic technologies, and to address the metrology needs of both the consumer and manufacturing sectors.

The National Institute of Standards and Technology is an agency of the U.S. Department of Commerce. 